\title{
Applications of Littlewood-Richardson tableaux to computing generic extension of semisimple invariant subspaces of nilpotent linear operators
}

\author{
Mariusz Kaniecki and Justyna Kosakowskad \\ Faculty of Mathematics and Computer Science, \\ Nicolaus Copernicus University \\ Chopina 12/18, 87-100 Toruń, Poland
}

MSC 2010: Primary: 47A15 Secondary: 05A17, 16G20, 16Z05

Key words: generic extensions, degenerations, partial orders, nilpotent operators, invariant subspaces, Littlewood-Richardson tableaux

\begin{abstract}
The main aim of the paper is to present a combinatorial algorithm that, applying Littlewood-Richardson tableaux with entries equal to 1 , computes generic extensions of semisimple invariant subspaces of nilpotent linear operators. Moreover, we discuss geometric properties of generic extensions and their connections with combinatorics.
\end{abstract}

\section{Introduction}

| The paper is motivated by results presented in [12, 13], where there are investigated relationships between Littlewood-Richardson tableaux and geometric properties of invariant subspaces of nilpotent linear operators. It is observed there that these relationships are deep and interesting. On the other hand, in [17], the existence of generic extensions for Dynkin quivers is proved and their connections with Hall algebras are investigated. Moreover,

\footnotetext{
E-mail addresses: kanies@mat.umk.pl (M.Kaniecki) justus@mat.umk.pl (J.Kosakowska)

This research did not receive any specific grant from funding agencies in the public, commercial, or not-for-profit sectors.
} 
by results presented in [4, 5, 6, 17] generic extensions of nilpotent linear operators exist and the operation of taking the generic extension provides the set of all isomorphism classes of nilpotent linear operators with a monoid structure. There are many results concerning this monoid and its properties (see [5, 6, 17, 11, 17]).

In the paper we investigate generic extensions of semisimple invariant subspaces of nilpotent linear operators. We show how combinatorial properties of Littlewood-Richardson tableaux are connected with geometric and algebraic properties of semisimple invariant subspaces. In particular, we present a combinatorial algorithm that, applying operations on LittlewoodRichardson tableaux with entries equal to 1, computes generic extensions of semisimple invariant subspaces of nilpotent linear operators.

Let $K$ be an algebraically closed field. By a nilpotent linear operator we mean a pair $\left(X_{2}, \varphi\right)$, where $X_{2}$ is a finite dimensional $K$-linear space and $\varphi: X_{2} \rightarrow X_{2}$ is a nilpotent $K$-linear endomorphism. An invariant subspace of a nilpotent linear operator $X_{2}=\left(X_{2}, \varphi\right)$ is a triple $\left(X_{1}, X_{2}, f\right)$, where $X_{1}$ is a linear space and $f: X_{1} \rightarrow X_{2}$ is a $k$-monomorphism such that $\varphi\left(f\left(X_{1}\right)\right) \subseteq f\left(X_{1}\right)$. An invariant subspace $\left(X_{1}, X_{2}, f\right)$ is said to be semisimple, if $\varphi\left(f\left(X_{1}\right)\right)=0$.

Let $a$ and $b$ be the $K$-dimensions of $X_{1}$ and $X_{2}$, respectively. Fix linear bases of $X_{1}$ and $X_{2}$. An invariant subspace $X=\left(X_{1}, X_{2}, f\right)$ one can interpret as the point $X=(f, \varphi)$ of the affine variety $\mathbb{M}_{b \times a}(K) \times \mathbb{M}_{b}(K)$, where $\mathbb{M}_{b \times a}(K)$ is the set of $b \times a$ matrices with coefficients in $k, \mathbb{M}_{b}(K)=\mathbb{M}_{b \times b}(K)$ and $\varphi, f$ are the matrices of $\varphi$ and $f$ in the bases fixed above (we use the same character for a map and its matrix). We also use the same character $X$ for an invariant subspace and for its corresponding point in the associated variety. Let ${ }^{a} V^{b} \subseteq \mathbb{M}_{b \times a}(K) \times \mathbb{M}_{b}(K)$ be the subset consisting of all points that correspond to semisimple invariant subspaces of nilpotent linear operators. The sets ${ }^{a} V^{b}$ are locally closed subsets of $\mathbb{M}_{b \times a}(K) \times \mathbb{M}_{b}(K)$ invariant under the action of the algebraic group $G=\operatorname{Gl}(a, b)=\operatorname{Gl}(a) \times \operatorname{Gl}(b)$, see Section 4 for details. Given a semisimple invariant subspace $X=\left(X_{1}, X_{2}, f\right)$ denote by $\mathcal{O}_{X}$ the orbit of $X$ in ${ }^{a} V^{b}$ under the action of $G$.

Given subsets $\mathcal{Y} \subseteq{ }^{a} V^{b}, \mathcal{X} \subseteq{ }^{c} V^{d}$, following [17], we define a subset

$$
\mathcal{E}(\mathcal{Y}, \mathcal{X}) \subseteq{ }^{a+c} V^{b+d}
$$

of all extensions of $Y \in \mathcal{Y}$ by $X \in \mathcal{X}$.

One of the main results of the paper is the following theorem. 
Theorem 1.1. (1) Given two semisimple invariant subspaces $X, Y$, the set $\mathcal{E}\left(\mathcal{O}_{Y}, \mathcal{O}_{X}\right)$ contains the unique dense orbit $\mathcal{O}_{Z}$. In particular $\overline{\mathcal{O}_{Z}}=\overline{\mathcal{E}\left(\mathcal{O}_{Y}, \mathcal{O}_{X}\right)}$, where $\overline{\mathcal{O}_{X}}$ denotes the Zariski closure of $\mathcal{O}_{X}$.

(2) The object $Z$ given in (1) is isomorphic with $Y * X$ constructed by Algorithm 5.2.

(3) $\overline{\mathcal{E}\left(\mathcal{O}_{Y}, \mathcal{O}_{X}\right)}=\mathcal{E}\left(\overline{\mathcal{O}_{Y}}, \overline{\mathcal{O}_{X}}\right)=\overline{\mathcal{O}_{Y * X}}$.

(4) The set $\overline{\mathcal{E}\left(\mathcal{O}_{Y}, \mathcal{O}_{X}\right)}=\mathcal{E}\left(\overline{\mathcal{O}_{Y}}, \overline{\mathcal{O}_{X}}\right)$ is irreducible.

The invariant subspace $Z=Y * X$ that correspond to the orbit $\mathcal{O}_{Z}$ given in Theorem 1.1 (1) is called the generic extension of $Y$ by $X$. In Section 5 we define (equivalently) $Y * X$ as an extension of $Y$ by $X$ with minimal dimension of its endomorphism ring. We present the proof in Sections 5 and 7 . The condition (1) of Theorem 1.1 is true for algebras of finite representation type, see [4, 17], while the conditions (3), (4) in general fail for this class of algebras, see [17, page 150, Remarks a)] for a counterexample. Therefore one can use the results of [4, Section 6], [17, Section 2] to prove the statement (1) easily. However, our proof of (1) and (2) is combinatorial and provides an algorithm that given $X, Y$ applying operations on Littlewood-Richardson tableaux computes $X * Y$.

In Section 3 we give a formula for dimension of an orbit $\mathcal{O}_{X}$, for given semisimple invariant subspace $X$. More precisely, we prove the following.

Theorem 1.2. Let $X \in{ }^{a} V^{b}$ and let $(\alpha, \beta, \gamma)$ be the triple of partitions associated with $X$, see Section 3 for details. We have

$$
\operatorname{dim} \mathcal{O}_{X}=b^{2}+a^{2}-n(\alpha)-n(\beta)-n(\gamma)-b
$$

where for a partition $\lambda$ we set $n(\lambda)=\sum_{i} \lambda_{i}(i-1)$.

The paper is organized as follows.

- In Section 2 we recall basic definitions, notation and facts concerning invariant subspaces.

- In Section 3 we prove Theorem 1.2.

- Section 4 contains description of three partial orders (i.e. $\leq_{\text {dom }}, \leq_{\text {hom }}$ and $\leq_{\text {deg }}$ ) defined on the set of isomorphism classes of semisimple invariant subspaces. In Theorem 4.4 we prove that these orders are equivalent. This result is used in the proof of Theorem 1.1. 
- In Sections [5] and 6 we present a combinatorial algorithm that computes generic extensions, illustrate it by examples and prove that the operation of taking the generic extension is associative. We also describe generators of the associative monoid of generic extensions.

- In Section 7 we discuss geometric properties of the set $\mathcal{E}(\mathcal{Y}, \mathcal{X})$ and we prove Theorem 1.1.

\section{Notation and definitions}

\subsection{Invariant subspaces}

Let $K$ be an arbitrary field and let $K[T]$ be the $K$-algebra of polynomials with one variable. It is well-known that a nilpotent linear operator $\left(X_{2}, \varphi\right)$ can be identified with the nilpotent $K[T]$-module $X_{2}$ via $T \cdot v=\varphi(v)$ for all $v \in X_{2}$, see [1, Chapter I, Example 2.6]. In the paper we will use this identification.

Let $\alpha=\left(\alpha_{1} \geq \ldots \geq \alpha_{n}\right)$ be a partition. We identify a partition with the corresponding Young diagram (parts of the partition corresponds to columns of its Young diagram). Denote by $|\alpha|=\alpha_{1}+\ldots+\alpha_{n}$ the length of $\alpha$ and by $\bar{\alpha}$ the partition conjugated to $\alpha$, i.e. $\bar{\alpha}$ is given by the Young diagram that is the transposition of the Young diagram of $\alpha$. Given partitions $\alpha, \beta$ we denote by $\alpha \cup \beta$ the union of these partitions, i.e. the multiset of parts of $\alpha \cup \beta$ is the union of multisets of parts of $\alpha$ and $\beta$.

For a partition $\alpha=\left(\alpha_{1} \geq \ldots \geq \alpha_{n}\right)$ we denote by $N_{\alpha}=N_{\alpha}(K)$ the nilpotent linear operator of type $\alpha$, i.e. the finite dimensional $K[T]$-module

$$
N_{\alpha}=N_{\alpha}(K)=K[T] /\left(T^{\alpha_{1}}\right) \oplus \ldots \oplus K[T] /\left(T^{\alpha_{n}}\right) .
$$

Note that the function $\alpha \mapsto N_{\alpha}$ defines a bijection between the set of all partitions and the set of all isomorphism classes of nilpotent linear operators. Since $T^{\alpha_{1}} N_{\alpha}=0$, the module $N_{\alpha}(K)$ has the natural $K[T] /\left(T^{\alpha_{1}}\right)$-module structure.

Consider the $k$-algebra

$$
\Lambda=\left(\begin{array}{cc}
K[T] & K[T] \\
0 & K[T]
\end{array}\right)
$$

and denote by $\bmod (\Lambda)$ the category of all finite dimensional right $\Lambda$-modules, and by $\bmod _{0}(\Lambda)$ the full subcategory of $\bmod (\Lambda)$ consisting of all modules 
for which the element $T=\left(\begin{array}{ll}T & 0 \\ 0 & T\end{array}\right)$ acts nilpotently. It is well known that objects of $\bmod _{0}(\Lambda)$ may be identified with systems $\left(N_{\alpha}, N_{\beta}, f\right)$, where $\alpha, \beta$ are partitions and $f: N_{\alpha} \rightarrow N_{\beta}$ is a $K[T]$-homomorphism. Let $X=\left(N_{\alpha}, N_{\beta}, f\right)$, $Y=\left(N_{\alpha^{\prime}}, N_{\beta^{\prime}}, f^{\prime}\right)$ be objects of $\bmod _{0}(\Lambda)$. A morphism $\Psi: X \rightarrow Y$ is a pair $\left(\psi_{1}, \psi_{2}\right)$, where $\psi_{1}: N_{\alpha} \rightarrow N_{\alpha^{\prime}}, \psi_{2}: N_{\beta} \rightarrow N_{\beta^{\prime}}$ are homomorphisms of $K[T]$-modules such that $f^{\prime} \psi_{1}=\psi_{2} f$, see [1, A.2, Example 2.7].

Denote by $\mathcal{S}$ or $\mathcal{S}(K)$ the full subcategory of $\bmod _{0}(\Lambda)$ consisting of all objects $N=\left(N_{\alpha}, N_{\beta}, f\right)$, where $f$ is a monomorphism. Note that objects of $\mathcal{S}$ may be identified with invariant subspaces of nilpotent linear operators.

For a natural number $n$, let $\mathcal{S}_{n}=\mathcal{S}_{n}(K)$ be the full subcategory of $\mathcal{S}$ consisting of all systems $\left(N_{\alpha}, N_{\beta}, f\right)$ such that $\alpha_{1} \leq n$.

It is easy to see that the objects in $\mathcal{S}_{1}$ are semisimple invariant subspaces.

We will denote by $\mathcal{S}_{a}^{b}$ the full subcategory of $\mathcal{S}_{1}$ consisting of all objects $\left(N_{\alpha}, N_{\beta}, f\right)$ such that $|\alpha|=a,|\beta|=b$. It is easy to see any object of $\mathcal{S}_{a}^{b}$ can be identified with a $\Lambda^{b}$-module, where

$$
\Lambda^{b}=\left(\begin{array}{cc}
K[T] /(T) & K[T] /(T) \\
0 & K[T] /\left(T^{b}\right)
\end{array}\right) .
$$

\subsection{Pickets}

The category $\mathcal{S}_{1}(K)$ is of particular interest for us in this paper. It has the discrete representation type (i.e. for any $d \in \mathbb{N}$ there is only finitely many isomorphism classes of objects of dimension $d$ ). Each indecomposable object is isomorphic to a picket that is, it has the form

$$
P_{0}^{m}=\left(0, N_{(m)}, 0\right)
$$

or

$$
P_{1}^{m}=\left(N_{(1)}, N_{(m)}, \iota\right)
$$

where $\iota(1)=T^{(m-1)}$, see [2]. Whenever we want to emphasize the dependence on the field $K$, we will write $P_{\ell}^{m}=P_{\ell}^{m}(K)$. It follows that for all natural numbers $a, b$ the category $\mathcal{S}_{a}^{b} \subseteq \mathcal{S}_{1}$ has finite representation type, i.e. there exists only finitely many isomorphism classes of objects in $\mathcal{S}_{a}^{b}$.

Thanks to this classification we can associate with any object $X$ of $\mathcal{S}_{1}(K)$ the LR-tableau $\Gamma(X)$ with entries equal to 1 (or the Pieri tableau). We prefer the name Littlewood-Richardson tableaux, because of their connections with invariant subspaces, see [15, 9, 12, 13]. Let $(\beta, \gamma)$ be a pair of partition. An 
LR-tableau of type $(\beta, \gamma)$ (or Pieri tableau) is a tableau $(\gamma \subseteq \beta)$ such that $\beta \backslash \gamma$ is a horizontal strip, i.e. $\gamma_{i} \leq \beta_{i} \leq \gamma_{i}+1$ for all $i$. In other words it is a skew diagram of shape $\beta \backslash \gamma$ with entries all equal to 1 . In the following table we list corresponding LR-tableaux for indecomposables.

\begin{tabular}{|c|c|c|}
\hline \multicolumn{4}{|c|}{ LR-tableaux for the indecomposable objects of $\mathcal{S}_{1}$} \\
\hline $\mathrm{X}$ & $P_{0}^{m}$ & $P_{1}^{m}$ \\
\hline & $\left(\begin{array}{c}\square \\
\vdots \\
\square\end{array}\right.$ & $m\left\{\begin{array}{c}\square \\
\vdots \\
\square \\
1\end{array}\right.$ \\
\hline
\end{tabular}

Let $X, X^{\prime}$ be objects of $\mathcal{S}_{1}$ and let $\beta, \gamma$ and $\beta^{\prime}, \gamma^{\prime}$ be partitions determining $\Gamma(X)$ and $\Gamma\left(X^{\prime}\right)$, respectively. The LR-tableau of the direct sum $X \oplus X^{\prime}$ is given by $\beta \cup \beta^{\prime}, \gamma \cup \gamma^{\prime}$. Recall that parts of a partition correspond to columns of its Young diagram.

Example 2.1. The object $X=P_{0}^{7} \oplus P_{1}^{7} \oplus P_{1}^{5} \oplus P_{1}^{2} \oplus P_{1}^{2} \oplus P_{0}^{1}$ has the following LR-tableau

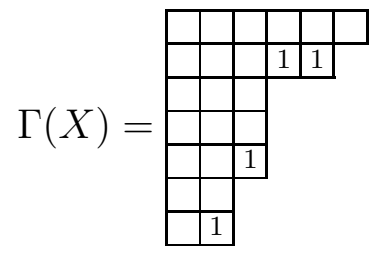

Note that the type of $\Gamma(X)$ is $(\beta, \gamma)$, where $\beta=(7,7,5,2,2,1)$ and $\gamma=$ $(7,6,4,1,1,1)$.

Remark 2.2. $\quad$ 1. The association $X \mapsto \Gamma(X)$ establishes a bijection between the set of all isomorphism classes of semisimple invariant subspaces and the set of all Littlewood-Richardson tableaux with entries equal to 1.

2. The invariant subspace $X$ is uniquely determined (up to isomorphism) by a pair of partitions $\gamma^{X} \subseteq \beta^{X}$, where $\beta^{X}$ is defined by the Young diagram of all boxes of $\Gamma(X)$ and $\gamma^{X}$ is given by the Young diagram of all empty boxes of $\Gamma(X)$.

3. If $X$ is given by $\gamma^{X} \subseteq \beta^{X}$, then the number of indecomposable direct summands of $X$ is equal to $\overline{\beta_{1}}$. 
For each pair $(X, Y)$ of indecomposable objects in $\mathcal{S}_{1}(K)$ we determine in the table below the dimension of the $K$-space $\operatorname{Hom}_{\mathcal{S}}(X, Y)$ of all $\mathcal{S}$-homomorphisms from $X$ to $Y$, see [20, Lemma 4] and [12].

\begin{tabular}{|c|c|c|}
\hline \multicolumn{3}{|c|}{ Dimensions of spaces $\operatorname{Hom}_{\mathcal{S}}(X, Y)$} \\
\hline$X$ & $Y=P_{0}^{m}$ & $P_{1}^{m}$ \\
\hline \hline$P_{0}^{\ell}$ & $\min \{\ell, m\}$ & $\min \{\ell, m\}$ \\
\hline$P_{1}^{\ell}$ & $\min \{\ell-1, m\}$ & $\min \{\ell, m\}$ \\
\hline
\end{tabular}

\section{Dimensions of orbits}

The main aim of this section is to prove Theorem 1.2 .

Assume that $K$ is an algebraically closed field. For natural numbers $a \leq b$ we consider the affine variety

$$
\mathbb{M}_{b \times a}(K) \times \mathbb{M}_{b}(K),
$$

where $\mathbb{M}_{b \times a}(K)$ is the set of $b \times a$ matrices with coefficients in $k$ and $\mathbb{M}_{b}(K)=$ $\mathbb{M}_{b \times b}(K)$. We work with the Zariski topology and with the induced topology for all subsets of $\mathbb{M}_{b \times a}(K) \times \mathbb{M}_{b}(K)$. We define ${ }^{a} V^{b}={ }^{a} V^{b}(K)$ as a subset of $\mathbb{M}_{b \times a}(K) \times \mathbb{M}_{b}(K)$ consisting of all points $X=(f, \varphi)$, such that $\varphi^{b}=$ $0, \varphi \cdot f=0$ and $f$ has maximal rank. On ${ }^{a} V^{b}$ the algebraic group $G=$ $\operatorname{Gl}(a, b)=\operatorname{Gl}(a) \times \mathrm{Gl}(b)$ acts via $(g, h) \cdot(f, \varphi)=\left(h f g^{-1}, h \varphi h^{-1}\right)$, where $\mathrm{Gl}(a)$ is the general linear group of all invertible $a \times a$ matrices. For a point $X \in{ }^{a} V^{b}$, denote by $\mathcal{O}_{X}$ the orbit of $X$ under the action of $G$ and by $G . X$ the stabilizer of $X$ in $G$.

Remark 3.1. $\quad$ 1. If $K$ is an algebraically closed field, then the set of points of ${ }^{a} V^{b}$ is in bijection with the set of objects of $\mathcal{S}_{a}^{b}$. This bijection is given by

$$
X=\left(N_{\alpha}, N_{\beta}, f\right) \longrightarrow X=\left(f, \varphi_{\beta}\right),
$$

where $N_{\beta}=\left(K^{b}, \varphi_{\beta}\right)$. We will identify an object $X$ of $\mathcal{S}_{a}^{b}$ with the corresponding point $X$ of ${ }^{a} V^{b}$.

2. Note that the $G$ - orbits in ${ }^{a} V^{b}$ are in 1-1-correspondence with the isomorphism classes of objects in $\mathcal{S}_{a}^{b}$. 
Proof of Theorem 1.2. Let $X=\left(N_{\alpha}, N_{\beta}, f\right) \in \mathcal{S}_{a}^{b}$, let $X=(f, \varphi) \in{ }^{a} V^{b}$ and let Coker $f \simeq N_{\gamma}$. Here the partition triple associated with $X$ is $(\alpha, \beta, \gamma)$.

It is well known that

$$
\operatorname{dim} \mathcal{O}_{X}=\operatorname{dim} \operatorname{Gl}(a, b)-\operatorname{dim} \operatorname{Gl}(a, b) \cdot X
$$

Moreover

$$
\operatorname{dim} \operatorname{Aut}_{\mathcal{S}}(X)=\operatorname{dim} \operatorname{Gl}(a, b) . X,
$$

where $\operatorname{Aut}_{\mathcal{S}}(X)$ is the group of $\mathcal{S}$-automorphisms of $X$. Since $\operatorname{dim} \operatorname{Gl}(a, b)=$ $a^{2}+b^{2}$, it is enough to prove that $\operatorname{dim} \operatorname{Aut}_{\mathcal{S}}(X)=n(\alpha)+n(\beta)+n(\gamma)+b$. The set $\operatorname{Aut}_{\mathcal{S}}(X)$ is dense and open in $\operatorname{End}_{\mathcal{S}}(X)$, and therefore $\operatorname{dim}_{\mathcal{S}} \operatorname{Aut}_{\mathcal{S}}(X)=$ $\operatorname{dim}_{K} \operatorname{End}_{\mathcal{S}}(X)$. Denote $[X, X]=\operatorname{dim}_{K} \operatorname{End}_{\mathcal{S}}(X)$. We prove that $[X, X]=$ $n(\alpha)+n(\beta)+n(\gamma)+b$ by the induction on the number $k$ of the indecomposable direct summands of $X$. If $k=1$, then $X \simeq P_{1}^{n}$ or $X \simeq P_{0}^{n}$. In both cases $n(\alpha)=n(\beta)=n(\gamma)=0, n=b$ and $[X, X]=n$. We are done.

Assume that $X \simeq \bigoplus_{i=1}^{k} P_{\varepsilon_{i}}^{\beta_{i}}$, where $k \geq 2, \varepsilon_{i} \in\{0,1\}$ for all $i$. Without loss of generality we can assume that $\beta_{1} \geq \ldots \geq \beta_{k}$ are numbered in such a way that for all $i$ :

$$
\text { if } \beta_{i}=\beta_{i+1} \text { and } \varepsilon_{i} \neq \varepsilon_{i+1} \text {, then } \varepsilon_{i}=0 \text { and } \varepsilon_{i+1}=1 \text {. }
$$

Let $X=X^{\prime} \oplus P_{\varepsilon_{k}}^{\beta_{k}}$, where $X^{\prime}=\bigoplus_{i=1}^{k-1} P_{\varepsilon_{i}}^{\beta_{i}}$ and let $s_{0}=\#\{i \in\{1, \ldots, k-$ 1\} ; $\left.\varepsilon_{i}=0\right\}, s_{1}=k-1-s_{0}$. Denote by $\left(\alpha^{\prime}, \beta^{\prime}, \gamma^{\prime}\right)$ the triple of partitions associated with $X^{\prime}$. Note that $n(\beta)=n\left(\beta^{\prime}\right)+(k-1) \beta_{k}$ and $b=|\beta|=\left|\beta^{\prime}\right|+\beta_{k}$.

First assume that $\varepsilon_{k}=1$. In this case $X=X^{\prime} \oplus P_{1}^{\beta_{k}}, n(\gamma)=n\left(\gamma^{\prime}\right)+(k-$ $1)\left(\beta_{k}-1\right)$ and $n(\alpha)=n\left(\alpha^{\prime}\right)+s_{1}$. Then (applying induction and data given in tables at the end of Section 2.2 ) we get

$$
\begin{gathered}
{[X, X]=\left[X^{\prime}, X^{\prime}\right]+\left[X, P_{1}^{\beta_{k}}\right]+\left[P_{1}^{\beta_{k}}, P_{1}^{\beta_{k}}\right]+\left[P_{1}^{\beta_{k}}, X\right]=} \\
n\left(\beta^{\prime}\right)+n\left(\gamma^{\prime}\right)+n\left(\alpha^{\prime}\right)+b^{\prime}+(k-1) \beta_{k}+\beta_{k}+s_{0}\left(\beta_{k}-1\right)+s_{1} \beta_{k}= \\
n(\beta)+b+n\left(\gamma^{\prime}\right)+(k-1)\left(\beta_{k}-1\right)+n\left(\alpha^{\prime}\right)+s_{1}=n(\alpha)+n(\beta)+n(\gamma)+b .
\end{gathered}
$$

Now, assume that $\varepsilon_{k}=0$. In this case $X=X^{\prime} \oplus P_{0}^{\beta_{k}}, n(\alpha)=n\left(\alpha^{\prime}\right)$ and $n(\gamma)=n\left(\gamma^{\prime}\right)+(k-1) \beta_{k}$, because of (3.4). Note that the property (3.4) implies that there is no direct summand of $X^{\prime}$ isomorphic with $P_{1}^{\beta_{k}}$ and that $\left[X, P_{0}^{\beta_{k}}\right]=(k-1) \beta_{k}$. Then (applying induction, data given in tables at the end of Section 2.2 and the property (3.4)) we get

$$
[X, X]=\left[X^{\prime}, X^{\prime}\right]+\left[P_{0}^{\beta_{k}}, X\right]+\left[P_{0}^{\beta_{k}}, P_{0}^{\beta_{k}}\right]+\left[X, P_{0}^{\beta_{k}}\right]=
$$




$$
\begin{gathered}
n\left(\beta^{\prime}\right)+n\left(\gamma^{\prime}\right)+n\left(\alpha^{\prime}\right)+b^{\prime}+(k-1) \beta_{k}+\beta_{k}+(k-1) \beta_{k}= \\
n(\beta)+b+n\left(\gamma^{\prime}\right)+(k-1) \beta_{k}+n(\alpha)=n(\alpha)+n(\beta)+n(\gamma)+b .
\end{gathered}
$$

Finally we get

$$
\operatorname{dim} \mathcal{O}_{X}=b^{2}+a^{2}-n(\alpha)-n(\beta)-n(\gamma)-b
$$

\section{Some partial orders in the category $\mathcal{S}_{1}$}

Fix integers $a, b$. We define three partial orders $\leq_{\text {dom }}, \leq_{\text {hom }}, \leq_{\text {deg }}$ on $\mathcal{S}_{b}^{a}$ and prove that they are equivalent. Results of this section are used in Section 5 and in the proof of Theorem 1.1 .

Definition 4.1. Let $X, Y \in \mathcal{S}_{b}^{a}$ be given by partitions $\gamma^{X} \subseteq \beta^{X}$ and $\gamma^{Y} \subseteq \beta^{Y}$, respectively.

- We say that $X, Y$ are in the dominance order, in symbols $X \leq_{\mathrm{dom}}$ $Y$, if partitions $\beta^{X}, \gamma^{X}$ and $\beta^{Y}, \gamma^{Y}$ are in the natural partial order, i.e. $\beta^{X} \leq_{\text {nat }} \beta^{Y}$ and $\gamma^{X} \leq_{\text {nat }} \gamma^{Y}$ (we say that partitions $\lambda$ and $\mu$ of the same length are in the natural order $\lambda \leq_{n a t} \mu$ if for any $k$ there is $\sum_{i=1}^{k} \lambda_{i} \geq \sum_{i=1}^{k} \mu_{i}$ or equivalently $\sum_{i=1}^{k} \bar{\lambda}_{i} \leq \sum_{i=1}^{k} \bar{\mu}_{i}$, where $\bar{\lambda}$ denotes the conjugate of $\lambda$ ).

- We say that $X, Y$ are in the hom order, in symbols $X \leq_{\text {hom }} Y$, if

$$
[Z, X] \leq[Z, Y]
$$

for any object $Z$ in $\mathcal{S}(K)$. Here we write $[X, Y]=\operatorname{dim}_{K} \operatorname{Hom}_{\mathcal{S}}(X, Y)$ for $\mathcal{S}$-objects $X, Y$.

Proposition 4.2. Let $K$ be an arbitrary field and let $X, Y$ be objects of $\mathcal{S}_{a}^{b}(K)$. The following conditions are equivalent:

(1) $X \leq_{\text {dom }} Y$,

(2) $[Z, X] \leq[Z, Y]$ for any object $Z$ in $\mathcal{S}_{1}(K)$,

(3) $X \leq$ hom $_{\text {h }}$ 
(4) $[Z, X] \leq[Z, Y]$ for any $\Lambda^{b+1}$-module $Z$,

(5) $[X, Z] \leq[Y, Z]$ for any $\Lambda^{b+1}$-module $Z$,

(6) $[Z, X] \leq[Z, Y]$ for any $\Lambda$-module $Z$.

Proof. The equivalence of (3) and (6) is proved in [12, Lemma 3.3 (2)]. Since assumptions of [12, Lemma 3.3 (2)] do not fit exactly to our situation, we rewrite this proof.

Any object $X=\left(X_{1}, X_{2}, f\right)$ of $\mathcal{S}$ is a $\Lambda$-module. Thus (6) implies (3). Now we prove that (3) implies (6). Assume that $\left[Z^{\prime}, X\right] \leq\left[Z^{\prime}, Y\right]$ for any object $Z^{\prime} \in \mathcal{S}$, we show that $[Z, X] \leq[Z, Y]$ for any $Z \in \bmod (\Lambda)$. Let $Z=\left(Z_{1}, Z_{2}, h\right) \in \bmod (\Lambda)$. Note that we can assume that $Z \in \bmod { }_{0}(\Lambda)$, because otherwise $[Z, X]=[Z, Y]=0$. Write $X=\left(X_{1}, X_{2}, f\right)$ where $f$ is a monomorphism. Consider the object $Z^{\prime}=\left(Z_{1}^{\prime}, Z_{2}, h^{\prime}\right) \in \mathcal{S}$, where $Z_{1}^{\prime}=$ $Z_{1} /$ Ker $h$ and $h^{\prime}: Z_{1}^{\prime} \rightarrow Z_{2}$ is induced by $h$. Let $a=\left(a_{1}, a_{2}\right): Z \rightarrow X$ be a morphism. Note that if $x \in \operatorname{Ker} h$, then $x \in \operatorname{Ker} a_{1}$ because $f$ is a monomorphism. Therefore there exists $a_{1}^{\prime}: Z_{1}^{\prime} \rightarrow X_{1}$ such that $a_{1}=$ $a_{1}^{\prime} \circ \mathrm{can}_{1}$, where $\operatorname{can}_{1}: Z_{1} \rightarrow Z_{1}^{\prime}$ is the canonical epimorphism. It is easy to see that the pair $a^{\prime}=\left(a_{1}^{\prime}, a_{2}\right)$ defines a morphism $Z^{\prime} \rightarrow Z$. Writing can $=\left(\operatorname{can}_{1}, 1\right): Z \rightarrow Z^{\prime}$, this morphism satisfies $a=a^{\prime} \circ$ can. Thus, can : $Z \rightarrow Z^{\prime}$ is a left approximation for $Z$ in $\mathcal{S}$. Since can is onto, it follows that $[Z, X]=\left[Z^{\prime}, Y\right]$.

Obviously, the condition (6) implies the conditions (2) and (4).

By [3, page 648], (4) and (5) are equivalent and by [13, Proposition 4.2], (3) implies (1).

The object $P_{1}^{0}=\left(N_{(1)}, 0,0\right)$ is the only indecomposable $\Lambda^{b+1}$-module that is not an object of $\mathcal{S}_{1}(K)$. Moreover $\left[X, P_{1}^{0}\right]=\left[Y, P_{1}^{0}\right]=a$. Therefore, by [13, Proposition 4.2] the condition (1) implies (5).

We prove that (4) implies (2). Assume that $[Z, X] \leq[Z, Y]$ for any $\Lambda^{b+1}$ module $Z$. We prove that $[Z, X] \leq[Z, Y]$ for any object $Z$ in $\mathcal{S}_{1}$. It is enough to prove this for indecomposable objects $Z=P_{0}^{m}$ and $Z=P_{1}^{m}$. Applying formulae for dimensions of homomorphisms spaces presented in Section 2.2 it is easy to see that for $i=1,0$ and $m>b$ we have $\left[P_{i}^{m}, X\right]=\left[P_{i}^{b+1}, X\right]$. This proves our claim, because $P_{i}^{b+1}$ is a $\Lambda^{b+1}$-module for $i=0,1$.

Next we show (2) implies (3). Assume that $\left[Z^{\prime \prime}, X\right] \leq\left[Z^{\prime \prime}, Y\right]$ for any object $Z^{\prime \prime}$ in $\mathcal{S}_{1}$. We prove that $\left[Z^{\prime}, X\right] \leq\left[Z^{\prime}, Y\right]$ for any object $Z^{\prime}$ in $\mathcal{S}$. Let $Z^{\prime}=\left(Z_{1}^{\prime}, Z_{2}^{\prime}, h^{\prime}\right) \in \mathcal{S}$ and $Y=\left(Y_{1}, Y_{2}, f\right) \in \mathcal{S}_{1}$, so $T \cdot Y_{1}=0$. Consider the object $Z^{\prime \prime}=\left(Z_{1}^{\prime \prime}, Z_{2}^{\prime \prime}, h^{\prime \prime}\right) \in \mathcal{S}_{1}$, where $Z_{1}^{\prime \prime}=Z_{1}^{\prime} /\left(T \cdot Z_{1}^{\prime}\right), Z_{2}^{\prime \prime}=Z_{2}^{\prime} / h^{\prime}\left(T \cdot Z_{1}^{\prime}\right)$ 
and $h^{\prime \prime}: Z_{1}^{\prime \prime} \rightarrow Z_{2}^{\prime \prime}$ is the map induced by $h^{\prime}$. Since $h^{\prime}$ is a monomorphism, so is $h^{\prime \prime}$. Let can $=\left(\operatorname{can}_{1}, \operatorname{can}_{2}\right): Z^{\prime} \rightarrow Z^{\prime \prime}$ be the canonical map. We show that a morphism $a=\left(a_{1}, a_{2}\right): Z^{\prime} \rightarrow Y$ factors over can. Since $T \cdot Y_{1}=0$, we have $T \cdot Z_{1}^{\prime} \subseteq \operatorname{Ker} a_{1}$, so $a_{1}$ factors over $\operatorname{can}_{1}$. We write $a_{1}=a_{1}^{\prime \prime} \circ$ can $_{1}$. Since $h^{\prime}\left(T \cdot Z_{1}^{\prime}\right) \subseteq \operatorname{Ker} a_{2}$, the map $a_{2}$ factors over $\operatorname{can}_{2}: Z_{2}^{\prime} \rightarrow Z_{2}^{\prime \prime}$, i.e. there is $a_{2}^{\prime \prime}$ with $a_{2}=a_{2}^{\prime \prime} \circ \operatorname{can}_{2}$. Since can ${ }_{1}$ is onto, the pair $a^{\prime \prime}=\left(a_{1}^{\prime \prime}, a_{2}^{\prime \prime}\right)$ is a morphism from $Z^{\prime \prime}$ to $Y$ in $\mathcal{S}$ satisfying $a=a^{\prime \prime} \circ$ can.

We have seen that can : $Z^{\prime} \rightarrow Z^{\prime \prime}$ is a left approximation for $Z^{\prime}$ in $\mathcal{S}_{1}$. Since can is onto, it follows that $\left[Z^{\prime}, Y\right]=\left[Z^{\prime \prime}, Y\right]$.

We are done.

Definition 4.3. Let $X=\left(N_{\alpha}, N_{\beta}, f\right)$ and $Y=\left(N_{\widetilde{\alpha}}, N_{\widetilde{\beta}}, g\right)$ be objects in $\mathcal{S}_{a}^{b}(K)$. The relation $X \leq_{\operatorname{deg}} Y$ holds if $\mathcal{O}_{Y} \subseteq \overline{\mathcal{O}_{X}}$ in ${ }^{a} V^{b}(K)$, where $\overline{\mathcal{O}_{X}}$ is the closure of $\mathcal{O}_{X}$.

Theorem 4.4. Let $K$ be an algebraically closed field and assume that $X, Y \in$ $\mathcal{S}_{a}^{b}(K)$. The following conditions are equivalent

1. $X \leq_{\text {dom }} Y$,

2. $X \leq_{\operatorname{deg}} Y$,

3. $X \leq_{\text {hom }} Y$.

Proof. Let $X, Y \in \mathcal{S}_{a}^{b}(K)$. Then $X, Y$ are $\Lambda^{b}$-modules.

If $K$ is an algebraically closed field, then by [21, Corollary, page 1314] we have

$$
X \leq_{\operatorname{deg}} Y \Longleftrightarrow X \leq_{\text {hom }} Y
$$

because the category $\mathcal{S}_{a}^{b}(K)$ (and the algebra $\Lambda^{b}$ ) is of finite representation type. Since by Proposition 4.2,

$$
X \leq_{\text {dom }} Y \Longleftrightarrow X \leq_{\text {hom }} Y
$$

we are done.

\section{Generic extensions - an algorithmic approach}

Let $K$ be an arbitrary field and let $X, Y \in \mathcal{S}_{1}(K)$. An object $Z \in \mathcal{S}_{1}$ is an extension of $Y$ by $X$ if there exists a short exact sequence of the form:

$$
0 \rightarrow X \rightarrow Z \rightarrow Y \rightarrow 0 .
$$


Note that the subcategory $\mathcal{S}_{1} \subseteq \mathcal{S}$ is not closed under extensions. In this paper we are interested only in the extensions that are objects of $\mathcal{S}_{1}$.

\subsection{Generic extensions - remarks}

If $K$ is an algebraically closed field, then one can define generic extensions in purely geometric way using Theorem 1.1 (1), see page 3. However, in view of the formula $\operatorname{dim} \mathcal{O}_{X}=\operatorname{dim} G-\operatorname{dim}_{K} \operatorname{End}_{\mathcal{S}}(X)$, we present the following equivalent definition.

Definition 5.1. The unique (up to isomorphism) extension $Z$ of $Y$ by $X$ with minimal $K$-dimension of the endomorphism ring $\operatorname{End}_{\mathcal{S}}(Z)$ is called the generic extension of $Y$ by $X$ and it is denoted by $Y * X$.

Generic extensions do not exist in general. The existence of generic extensions in the category $\mathcal{S}_{1}$ one can prove applying geometric arguments, see remarks on page 3 below Theorem 1.1. In this section we present a combinatorial algorithm that given objects $X, Y \in \mathcal{S}_{1}$ computes the generic extension $Y * X$. This in fact give an independent combinatorial proof of Theorem 1.1 (1) and (2).

To prove the correctness of Algorithm 5.2 we have to show that $Y * X$ computed by this algorithm is an extension of $Y$ by $X$ (which is done in Lemma 5.7) and that $Y * X$ is the unique extension of $Y$ by $X$ with minimal dimension of the endomorphism ring (which is done in Corollary 5.12). The proof of Corollary 5.12 uses Lemma 5.10, where we show that the extension of $Y$ by $X$ computed by Algorithm 5.2 is a minimal extension in the order $\leq_{\text {dom }}$ (and hence in $\leq_{\text {deg }}$, by Theorem 4.4).

\subsection{Algorithm}

The following algorithm computes the generic extension in the category $\mathcal{S}_{1}$ (the proof is presented in next subsections). For an object $X \in \mathcal{S}_{1}$, by $\gamma^{X} \subseteq \beta^{X}$ we denote the pair of partitions uniquely (up to isomorphism) defining $X$. We identify $X$ with the pair $\left(\gamma^{X}, \beta^{X}\right)$.

Algorithm 5.2. Input. $X, Y \in \mathcal{S}_{1}$.

Output. The generic extension $Z=Y * X \in \mathcal{S}_{1}$ of $Y$ by $X$.

1. $\gamma^{Z}=\gamma^{X}+\gamma^{Y}$ 
2. set $n=0$

3. for any $i=1, \ldots, \min \left\{\overline{\beta_{1}^{X}}, \overline{\beta_{1}^{Y}}\right\}$, do

(a) put $\beta_{i}^{Z}=\beta_{i}^{X}+\gamma_{i}^{Y}$

(b) if $\beta_{i}^{Y} \neq \gamma_{i}^{Y}$, then put $n=n+1$

4. if $\overline{\beta_{1}^{X}}>\min \left\{\overline{\beta_{1}^{X}}, \overline{\beta_{1}^{Y}}\right\}$, then for $i=\min \left\{\overline{\beta_{1}^{X}}, \overline{\beta_{1}^{Y}}\right\}+1, \ldots, \overline{\beta_{1}^{X}}$ put

$$
\beta_{i}^{Z}=\beta_{i}^{X},
$$

else for $i=\min \left\{\overline{\beta_{1}^{X}}, \overline{\beta_{1}^{Y}}\right\}+1, \ldots, \overline{\beta_{1}^{Y}}$ we set

$$
\begin{gathered}
\beta_{i}^{Z}=\beta_{i}^{Y}+\mathbf{1}\left\{\gamma_{i}^{Y}=\beta_{i}^{Y} \text { and } n>0\right\} \\
n=n-1\left\{\gamma_{i}^{Y}=\beta_{i}^{Y} \text { and } n>0\right\}
\end{gathered}
$$

where

$$
\mathbf{1}\left\{\gamma_{i}^{Y}=\beta_{i}^{Y} \text { and } n>0\right\}= \begin{cases}1 & \text { if } \gamma_{i}^{Y}=\beta_{i}^{Y} \text { and } n>0 \\ 0 & \text { otherwise }\end{cases}
$$

5. We set

$$
\beta^{Z}=\beta^{Z} \cup \alpha
$$

where $\alpha=(1,1, \ldots, 1)$ is a partition with $n$ copies of 1 .

We illustrate this algorithm by the following examples.

Example 5.3. Let $X=P_{0}^{4} \oplus P_{1}^{4} \oplus P_{1}^{3}$ and $Y=P_{1}^{4} \oplus P_{0}^{3} \oplus P_{0}^{2} \oplus P_{1}^{2} \oplus P_{0}^{1} \oplus P_{0}^{1}$. Note that

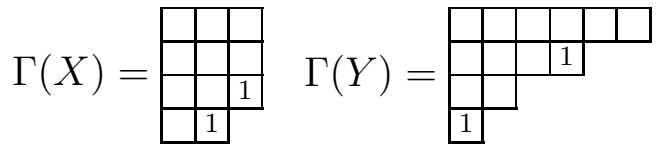

and $\beta^{X}=(4,4,3), \gamma^{X}=(4,3,2), \beta^{Y}=(4,3,2,2,1,1), \gamma^{Y}=(3,3,2,1,1,1)$. The Step 1 of Algorithm 5.2 gives

$$
\gamma^{Z}=\gamma^{X}+\gamma^{Y}=(7,6,4,1,1,1) .
$$

In our case $\min \left\{\overline{\beta_{1}^{X}}, \overline{\beta_{1}^{Y}}\right\}=3$ and therefore, for $i=1,2,3$, the Step 3 of Algorithm 5.2 sets $\beta_{i}^{Z}=\beta_{i}^{X}+\gamma_{i}^{Y}$. We have

$$
\beta_{1}^{Z}=7, \beta_{2}^{Z}=7, \beta_{3}^{Z}=5 \text {. }
$$


Note that $n=1$.

For $i>3=\overline{\beta_{1}^{X}}=\min \left\{\overline{\beta_{1}^{X}}, \overline{\beta_{1}^{Y}}\right\}$ the Step 4 of the algorithm gives

$$
\beta_{i}^{Z}=\beta_{i}^{Y}+\mathbf{1}\left\{\gamma_{i}^{Y}=\beta_{i}^{Y} \text { and } n>0\right\}, \quad n=n-\mathbf{1}\left\{\gamma_{i}^{Y}=\beta_{i}^{Y} \text { and } n>0\right\}
$$

For $i=4$ we have:

$$
\beta_{4}^{Z}=\beta_{4}^{Y}=2 \text { and } n=1 \text {, because } \gamma_{4}^{Y} \neq \beta_{4}^{Y},
$$

and for $i=5$ :

$$
\beta_{5}^{Z}=\beta_{5}^{Y}+1=2 \text { and } n=0 \text {, because } \gamma_{5}^{Y}=\beta_{5}^{Y} .
$$

Now $n=0$, and therefore for $i=6$ we get:

$$
\beta_{6}^{Y}=1
$$

Finally, we get $\beta^{Z}=(7,7,5,2,2,1)$ and $\gamma^{Z}=(7,6,4,1,1,1)$, then

$$
Z=P_{0}^{7} \oplus P_{1}^{7} \oplus P_{1}^{5} \oplus P_{1}^{2} \oplus P_{1}^{2} \oplus P_{0}^{1}
$$

and LR-tableau of $Z$ has the form (by the gray colour we marked part of the diagram corresponding to the object $Y$ ):

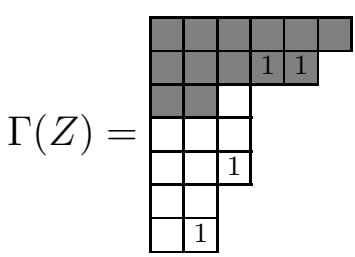

Example 5.4. Let $X=P_{1}^{4} \oplus P_{0}^{3} \oplus P_{0}^{2} \oplus P_{1}^{2} \oplus P_{0}^{1} \oplus P_{0}^{1}$ and $Y=P_{0}^{4} \oplus P_{1}^{4} \oplus P_{1}^{3}$. Then $\beta^{X}=(4,3,2,2,1,1), \gamma^{X}=(3,3,2,1,1,1)$ and $\beta^{Y}=(4,4,3), \gamma^{Y}=$ $(4,3,2)$ and $\min \left\{\overline{\beta_{1}^{X}}, \overline{\beta_{1}^{Y}}\right\}=3$. The Step 1 of Algorithm 5.2 gets

$$
\gamma^{Z}=\gamma^{X}+\gamma^{Y}=(7,6,4,1,1,1) .
$$

For $i=1,2,3$, the Step 3 of the Algorithm sets $\beta_{i}^{Z}=\beta_{i}^{X}+\gamma_{i}^{Y}$ so we have

$$
\beta_{1}^{Z}=8, \beta_{2}^{Z}=6, \beta_{3}^{Z}=4
$$

moreover $n=2$. 
For $\overline{\beta_{1}^{X}} \geq i>3=\overline{\beta_{1}^{Y}}=\min \left\{\overline{\beta_{1}^{X}}, \overline{\beta_{1}^{Y}}\right\}$ the Step 4 of the Algorithm sets

$$
\beta_{i}^{Z}=\beta_{i}^{X} \text {. }
$$

We have:

$$
\beta_{4}^{Z}=2, \beta_{5}^{Z}=1, \beta_{6}^{Z}=1 \text {. }
$$

Parts of the partition $\beta^{Z}$ for $i=1,2, \ldots, \max \left\{\overline{\beta_{1}^{X}}, \overline{\beta_{1}^{Y}}\right\}$ are constructed, but $n=2 \neq 0$. Therefore the Step 5 of Algorithm 5.2 gives $\beta^{Z}=\beta^{Z} \cup(1,1)$. Finally, we get $\beta^{Z}=(8,6,4,2,1,1,1,1)$ and $\gamma^{Z}=(7,6,4,1,1,1)$, so

$$
Z=P_{1}^{8} \oplus P_{0}^{6} \oplus P_{0}^{4} \oplus P_{1}^{2} \oplus P_{0}^{1} \oplus P_{0}^{1} \oplus P_{1}^{1} \oplus P_{1}^{1}
$$

and LR-tableau of $Z$ has the following form (by the gray colour we marked part of the diagram corresponding to the object $Y$ ):

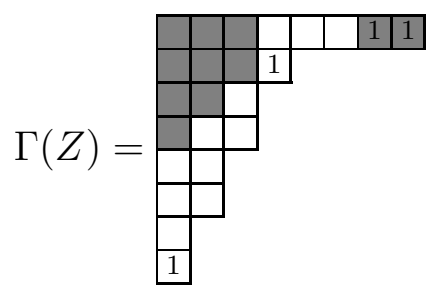

Example 5.5. Let $X=P_{1}^{2}$ and $Y=P_{0}^{1} \oplus P_{0}^{1} \oplus P_{1}^{1}$. Note that

$$
\Gamma(X)=\square \quad \Gamma(Y)=\square \square 1
$$

and $\beta^{X}=(2), \gamma^{X}=(1), \beta^{Y}=(1,1,1), \gamma^{Y}=(1,1)$. Note that, for $Z=X * Y$, we get $\beta^{Z}=(2,1,1,1)$ and $\gamma^{Z}=(2,1), Z=P_{0}^{2} \oplus P_{0}^{1} \oplus P_{1}^{1} \oplus P_{1}^{1}$ and LR-tableau of $Z$ has the form:

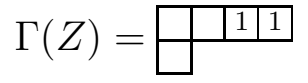

For $Z^{\prime}=Y * X$, we get $\beta^{Z^{\prime}}=(3,1,1), \gamma^{Z^{\prime}}=(2,1), Z=P_{1}^{3} \oplus P_{0}^{1} \oplus P_{1}^{1}$ and LR-tableau of $Z^{\prime}$ has the form:

$$
\Gamma\left(Z^{\prime}\right)=\begin{array}{|l|l|}
\hline & 11 \\
\hline 1 &
\end{array}
$$

Example 5.6. Let $X=P_{1}^{2}$ and $Y=P_{1}^{2} \oplus P_{0}^{1} \oplus P_{0}^{1} \oplus P_{1}^{1}$. Note that

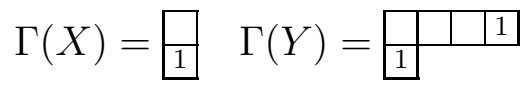


and $\beta^{X}=(2), \gamma^{X}=(1), \beta^{Y}=(2,1,1,1), \gamma^{Y}=(1,1,1)$. Note that, for $Z=$ $X * Y$, we get $\beta^{Z}=(3,1,1,1,1), \gamma^{Z}=(2,1,1), Z=P_{1}^{3} \oplus P_{0}^{1} \oplus P_{0}^{1} \oplus P_{1}^{1} \oplus P_{1}^{1}$ and

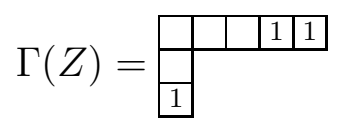

For $Z^{\prime}=Y * X$, we get $\beta^{Z^{\prime}}=(3,2,1,1), \gamma^{Z^{\prime}}=(2,1,1), Z=P_{1}^{3} \oplus P_{1}^{2} \oplus P_{0}^{1} \oplus P_{1}^{1}$ and

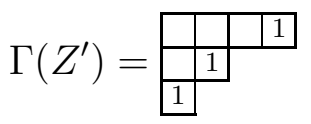

\subsection{Proof of correctness of Algorithm 5.2}

The following lemma shows that Algorithm 5.2 computes an extension of $Y$ by $X$.

Lemma 5.7. Let $X, Y \in \mathcal{S}_{1}$. The object $Z$ constructed by Algorithm 5.2 is an extension of $Y$ by $X$.

Proof. For natural numbers $m, r, k$ such that $m \geq 1$ and $r>k$ let

$$
E_{1}(m, r, k): \quad 0 \rightarrow P_{0}^{m} \rightarrow P_{0}^{m+r-1} \oplus P_{1}^{k+1} \rightarrow P_{1}^{r} \oplus P_{0}^{k} \rightarrow 0
$$

denotes the following short exact sequence:

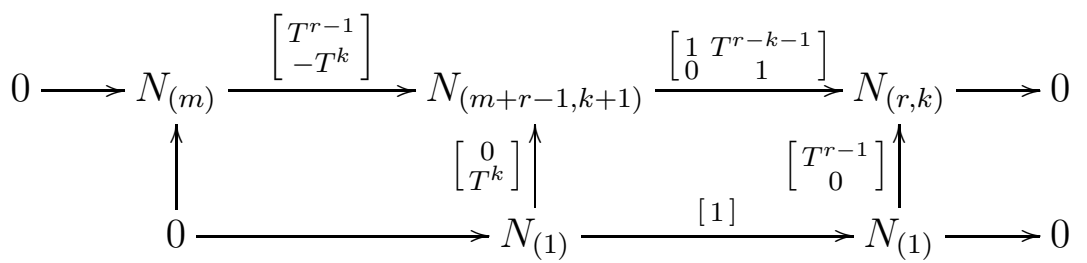

and let

$$
E_{2}(m, r, k): \quad 0 \rightarrow P_{1}^{m} \rightarrow P_{1}^{m+r-1} \oplus P_{1}^{k+1} \rightarrow P_{1}^{r} \oplus P_{0}^{k} \rightarrow 0
$$

for $m \geq 2$ denotes the following short exact sequence

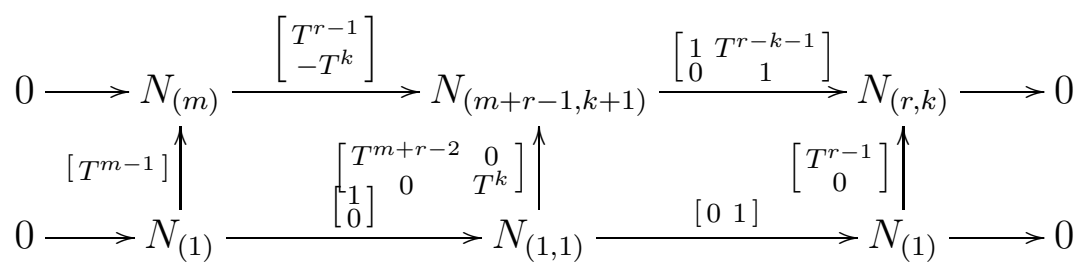


and for $m=1$ the following one

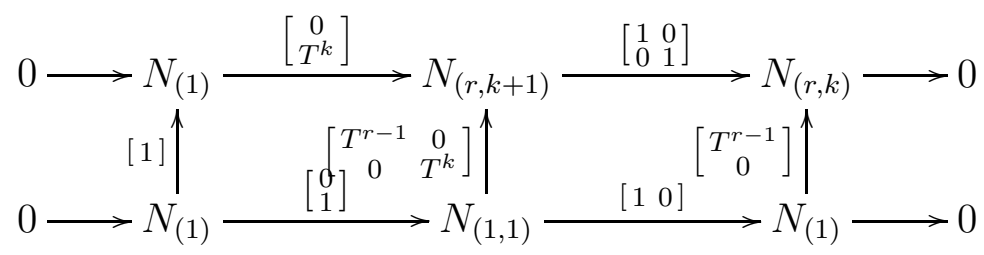

We fix $X, Y \in \mathcal{S}_{1}$. Consider the following cases.

Case 1. Assume that $\overline{\beta_{1}^{X}}=\overline{\beta_{1}^{Y}}$, i.e. the objects $X$ and $Y$ have the same number of indecomposable direct summands. Note that in this case the Step 4 of the algorithm does not hold (because the loops start from $\left.\min \left\{\overline{\beta_{1}^{X}}, \overline{\beta_{1}^{Y}}\right\}+1\right)$. Moreover since $X, Y \in \mathcal{S}_{1}$, for any $i$ we have: if $\beta_{i}^{*}>\gamma_{i}^{*}$, then $\beta_{i}^{*}=\gamma_{i}^{*}+1$, where $* \in\{X, Y\}$.

It is easy to see that in this case the object $Z$ constructed by the algorithm is the direct sum of the middle terms of the following short exact sequences $\left(i=1, \ldots, \min \left\{\overline{\beta_{1}^{X}}, \overline{\beta_{1}^{Y}}\right\}\right)$ :

$$
0 \rightarrow P_{0}^{\beta_{i}^{X}} \rightarrow P_{0}^{\beta_{i}^{X}+\beta_{i}^{Y}} \rightarrow P_{0}^{\beta_{i}^{Y}} \rightarrow 0
$$

if $\beta_{i}^{X}=\gamma_{i}^{X}$ and $\beta_{i}^{Y}=\gamma_{i}^{Y}$;

$$
0 \rightarrow P_{1}^{\beta_{i}^{X}} \rightarrow P_{1}^{\beta_{i}^{X}+\beta_{i}^{Y}} \rightarrow P_{0}^{\beta_{i}^{Y}} \rightarrow 0
$$

if $\beta_{i}^{X}>\gamma_{i}^{X}$ and $\beta_{i}^{Y}=\gamma_{i}^{Y}$;

$$
E_{1}\left(\beta_{i}^{X}, \beta_{i}^{Y}, 0\right): \quad 0 \rightarrow P_{0}^{\beta_{i}^{X}} \rightarrow P_{0}^{\beta_{i}^{X}+\gamma_{i}^{Y}} \oplus P_{1}^{1} \rightarrow P_{1}^{\beta_{i}^{Y}} \rightarrow 0,
$$

if $\beta_{i}^{X}=\gamma_{i}^{X}$ and $\beta_{i}^{Y}>\gamma_{i}^{Y}$;

$$
E_{2}\left(\beta_{i}^{X}, \beta_{i}^{Y}, 0\right): \quad 0 \rightarrow P_{1}^{\beta_{i}^{X}} \rightarrow P_{1}^{\beta_{i}^{X}+\gamma_{i}^{Y}} \oplus P_{1}^{1} \rightarrow P_{1}^{\beta_{i}^{Y}} \rightarrow 0,
$$

if $\beta_{i}^{X}>\gamma_{i}^{X}$ and $\beta_{i}^{Y}>\gamma_{i}^{Y}$.

Case 2. Assume that $\overline{\beta_{1}^{X}}>\overline{\beta_{1}^{Y}}$, i.e. the object $X$ has more indecomposable direct summands than $Y$. It is easy to see that in this case the object $Z$ constructed by the algorithm is the direct sum of the middle terms of the short exact sequences given in Case 1 for $i=1, \ldots, \min \left\{\overline{\beta_{1}^{X}}, \overline{\beta_{1}^{Y}}\right\}$ and the short exact sequences (for $i=\min \left\{\overline{\beta_{1}^{X}}, \overline{\beta_{1}^{Y}}\right\}+1, \ldots, \overline{\beta_{1}^{X}}$ ):

$$
0 \rightarrow P_{1}^{\beta_{i}^{X}} \rightarrow P_{1}^{\beta_{i}^{X}} \rightarrow 0 \rightarrow 0
$$


if $\beta_{i}>\gamma_{i}$;

$$
0 \rightarrow P_{0}^{\beta_{i}^{X}} \rightarrow P_{0}^{\beta_{i}^{X}} \rightarrow 0 \rightarrow 0
$$

if $\beta_{i}=\gamma_{i}$.

Case 3. Assume that $\overline{\beta_{1}^{X}}<\overline{\beta_{1}^{Y}}$, i.e. the object $X$ has less indecomposable direct summands than $Y$.

Let

$$
\begin{gathered}
I=\left\{i \in\left\{1, \ldots, \overline{\beta_{1}^{X}}\right\} ; \beta_{i}^{Y}>\gamma_{i}^{Y}\right\}=\left\{i_{1}, \ldots, i_{n}\right\} \\
J=\left\{j=\overline{\beta_{1}^{X}}+1, \ldots, \overline{\beta_{1}^{Y}} ; \beta_{j}^{Y}=\gamma_{j}^{Y}\right\}=\left\{j_{1}, \ldots, j_{|J|}\right\}
\end{gathered}
$$

and we set the numeration as follows $j_{i}<j_{i+1}$ for all $i$.

Let $\bar{I}=\left\{i_{1}, \ldots, i_{\min \{n,|J|\}}\right\}$.

In this case the object $Z$ constructed by the algorithm is the direct sum of the middle terms of the following short exact sequences $\left(i=1, \ldots, \min \left\{\overline{\beta_{1}^{X}}, \overline{\beta_{1}^{Y}}\right\}\right)$ :

$$
0 \rightarrow P_{0}^{\beta_{i}^{X}} \rightarrow P_{0}^{\beta_{i}^{X}+\beta_{i}^{Y}} \rightarrow P_{0}^{\beta_{i}^{Y}} \rightarrow 0
$$

if $\beta_{i}^{X}=\gamma_{i}^{X}$ and $\beta_{i}^{Y}=\gamma_{i}^{Y}$;

$$
0 \rightarrow P_{1}^{\beta_{i}^{X}} \rightarrow P_{1}^{\beta_{i}^{X}+\beta_{i}^{Y}} \rightarrow P_{0}^{\beta_{i}^{Y}} \rightarrow 0
$$

if $\beta_{i}^{X}>\gamma_{i}^{X}$ and $\beta_{i}^{Y}=\gamma_{i}^{Y}$;

$$
E_{1}\left(\beta_{i_{s}}^{X}, \beta_{i_{s}}^{Y}, \beta_{j_{s}}^{Y}\right): \quad 0 \rightarrow P_{0}^{\beta_{i}^{X}} \rightarrow P_{0}^{\beta_{i}^{Z}+\gamma_{i}^{Y}} \oplus P_{1}^{\beta_{j_{s}}^{Y}+1} \rightarrow P_{1}^{\beta_{i}^{Y}} \oplus P_{0}^{\beta_{j_{s}}^{Y}} \rightarrow 0
$$

if $\beta_{i}^{X}=\gamma_{i}^{X}, \beta_{i}^{Y}>\gamma_{i}^{Y}$ and $i=i_{s} \in \bar{I}$

$$
E_{2}\left(\beta_{i_{s}}^{X}, \beta_{i_{s}}^{Y}, \beta_{j_{s}}^{Y}\right): \quad 0 \rightarrow P_{1}^{\beta_{i}^{X}} \rightarrow P_{1}^{\beta_{i}^{Z}+\gamma_{i}^{Y}} \oplus P_{1}^{\beta_{j_{s}}^{Y}+1} \rightarrow P_{1}^{\beta_{i}^{Y}} \oplus P_{0}^{\beta_{j_{s}}^{Y}} \rightarrow 0
$$

if $\beta_{i}^{X}>\gamma_{i}^{X}, \beta_{i}^{Y}>\gamma_{i}^{Y}$ and $i=i_{s} \in \bar{I}$.

The following two cases holds if the number $n$ is reduced to 0 in the Step 4 of Algorithm 5.2 ,

$$
0 \rightarrow 0 \rightarrow P_{1}^{\beta_{i}^{Y}} \rightarrow P_{1}^{\beta_{i}^{Y}} \rightarrow 0
$$

if $\beta_{i}>\gamma_{i}$ and $i>\overline{\beta_{1}^{X}}$

$$
0 \rightarrow 0 \rightarrow P_{0}^{\beta_{i}^{Y}} \rightarrow P_{0}^{\beta_{i}^{Y}} \rightarrow 0
$$


if $\beta_{i}=\gamma_{i}$ and $i \in\left\{j_{\min \{n,|J|\}+1}, \ldots, j_{|J|}\right\}$.

Otherwise the 5th Step of Algorithm is execute, the following two cases illustrated this situation:

$$
E_{1}\left(\beta_{i}^{X}, \beta_{i}^{Y}, 0\right): \quad 0 \rightarrow P_{0}^{\beta_{i}^{X}} \rightarrow P_{0}^{\beta_{i}^{Z}+\gamma_{i}^{Y}} \oplus P_{1}^{1} \rightarrow P_{1}^{\beta_{i}^{Y}} \rightarrow 0,
$$

if $\beta_{i}^{X}=\gamma_{i}^{X}, \beta_{i}^{Y}>\gamma_{i}^{Y}$ and $i \in I \backslash \bar{I}$;

$$
E_{2}\left(\beta_{i}^{X}, \beta_{i}^{Y}, 0\right): \quad 0 \rightarrow P_{1}^{\beta_{i}^{X}} \rightarrow P_{1}^{\beta_{i}^{Z}+\gamma_{i}^{Y}} \oplus P_{1}^{1} \rightarrow P_{1}^{\beta_{i}^{Y}} \rightarrow 0,
$$

if $\beta_{i}^{X}>\gamma_{i}^{X}, \beta_{i}^{Y}>\gamma_{i}^{Y}$ and $i \in I \backslash \bar{I}$.

The lemma is proved.

The following two lemmata are used in the proof of Lemma 5.10.

Lemma 5.8. Let $X=\left(N_{\alpha^{X}}, N_{\beta^{X}}, f_{X}\right), Y=\left(N_{\alpha^{Y}}, N_{\beta^{Y}}, f_{Y}\right) \in \mathcal{S}_{1}$. If an object $Z \in \mathcal{S}_{1}$ is an extension of $Y$ by $X$, then $Z$ is also an extension of $\widetilde{Y}$ by $\widetilde{X}$, where $\widetilde{X}=\left(N_{\alpha^{X}} \oplus N_{\alpha^{Y}}, N_{\beta^{X}} \oplus N_{\alpha^{Y}}, f_{X} \times i d_{N_{\alpha} Y}\right)$ and $\widetilde{Y}=\left(0, N_{\gamma^{Y}}, 0\right)$ for $\gamma^{Y}$ such that $\operatorname{Coker} f_{Y} \simeq N_{\gamma^{Y}}$.

Proof. If $Z$ is an extension of $Y$ by $X$, then there exists a commutative diagram with exact rows:

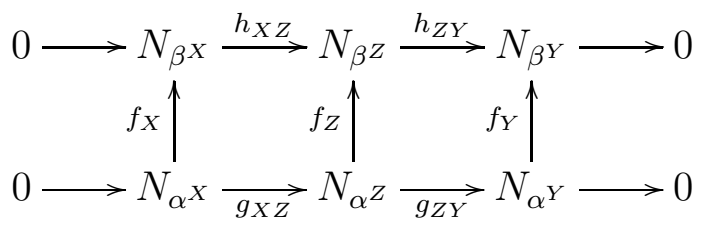

Since the morphisms $f_{X}, f_{X}, f_{Y}$ are injective, this diagram induces the following commutative diagram with exact rows and columns. 


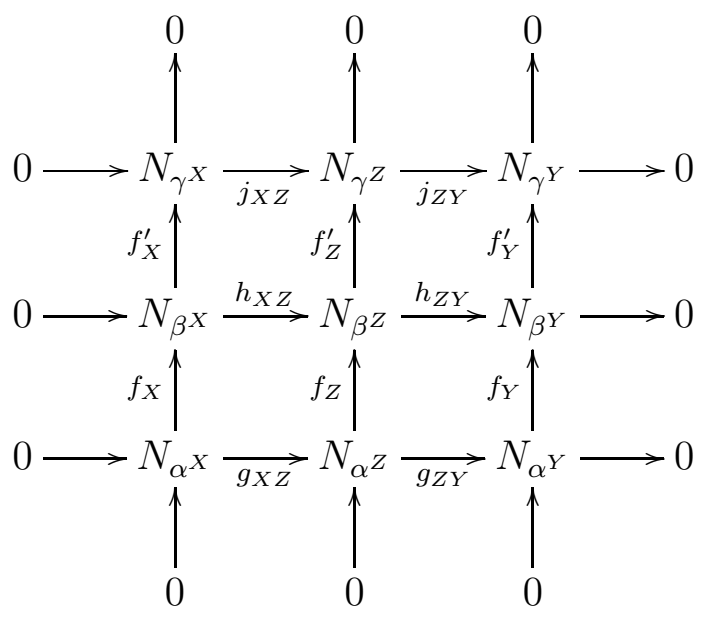

Since we are working in the category $\mathcal{S}_{1}$ the bottom row splits (because it contains only semisimple objects). Therefore there exists $g_{Z Y}^{\prime}: N_{\alpha^{Y}} \rightarrow N_{\alpha^{Z}}$ such that $g_{Z Y} \circ g_{Z Y}^{\prime}=\operatorname{id}_{N_{\alpha} Y}$. We consider the following diagram:

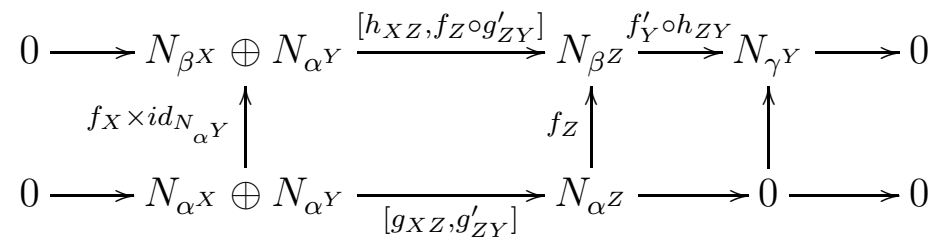

It is straightforward to check that this diagram is commutative. Since $N_{\alpha^{X}} \oplus N_{\alpha^{Y}}$ and $N_{\alpha^{Z}}$ are semisimple nilpotent operators with the same dimension, the bottom row is exact. Moreover $h_{Z Y} \circ f_{Z} \circ g_{Z Y}^{\prime}=f_{Y}$ is injective. It follows that $0=\left(f_{Z} \circ g_{Z Y}^{\prime}\left(N_{\alpha^{X}}\right)\right) \cap \operatorname{Ker} h_{Z Y}=\left(f_{Z} \circ g_{Z Y}^{\prime}\left(N_{\alpha^{X}}\right)\right) \cap \operatorname{Im} h_{X Z}$. Now, it is easy to prove that the morphism $\left[h_{X Z}, f_{Z} \circ g_{Z Y}^{\prime}\right]$ is injective and the top row is exact. This finishes the proof.

The following fact is proved in [11, Lemma 3.3].

Lemma 5.9. Let $\sigma, \nu, \mu$ be partitions. If there exists a short exact sequence:

$$
0 \rightarrow N_{\nu} \rightarrow N_{\sigma} \rightarrow N_{\mu} \rightarrow 0
$$

then for any $m \geq 1$ :

$$
\sum_{i=1}^{m} \sigma_{i} \leq \sum_{i=1}^{m} \lambda_{i},
$$

where $\lambda=\nu+\mu$. 
In the following lemma we prove that the extension computed by Algorithm 5.2 is minimal in the dominance order $\leq_{\text {dom }}$.

Lemma 5.10. If $\widetilde{Z}$ is the extension of $Y$ by $X$ constructed by Algorithm 5.2 , then for any extension $Z$ of $Y$ by $X$ and for any $j \geq 1$ we have $\sum_{i=1}^{j} \gamma_{j}^{\widetilde{Z}} \geq \sum_{i=1}^{j} \gamma_{j}^{Z}$ and $\sum_{j=1}^{i} \beta_{j}^{\widetilde{Z}} \geq \sum_{j=1}^{i} \beta_{j}^{Z}$. In particular $\widetilde{Z} \leq_{\operatorname{dom}} Z$.

Proof. Let $Z$ be an arbitrary extension of $Y$ by $X$. It induces short exact sequences:

$$
\begin{aligned}
& 0 \rightarrow N_{\beta^{X}} \rightarrow N_{\beta^{Z}} \rightarrow N_{\beta^{Y}} \rightarrow 0 \\
& 0 \rightarrow N_{\gamma^{X}} \rightarrow N_{\gamma^{Z}} \rightarrow N_{\gamma^{Y}} \rightarrow 0
\end{aligned}
$$

Applying Algorithm 5.2 to $X$ and $Y$ we compute $\widetilde{Z}$. Note that $\gamma^{\widetilde{Z}}=$ $\gamma^{X}+\gamma^{Y}$. By Lemma 5.9, for all $i$, we get:

$$
\sum_{j=1}^{i} \gamma_{j}^{Z} \leq \sum_{j=1}^{i} \gamma_{j}^{\widetilde{Z}}
$$

To prove the second part of lemma we consider $Z$ as an extension of $\widetilde{Y}=\left(0, N_{\gamma^{Y}}, 0\right)$ by $\widetilde{X}=\left(N_{\alpha^{X}} \oplus N_{\alpha^{Y}}, N_{\beta^{X}} \oplus N_{\alpha^{Y}}, f_{\widetilde{X}}\right)$. It is possible by Lemma 5.8.

For all $i=1, \ldots, \min \left\{\overline{\beta_{1}^{X}}, \overline{\beta_{1}^{Y}}\right\}$, by the Step 3 (a) of Algorithm [5.2, we have $\beta_{i}^{\widetilde{Z}}=\beta_{i}^{X}+\gamma_{i}^{Y}$. Since the number of indecomposable direct summands of $N_{\beta^{X}}$ is equal to $\overline{\beta_{1}^{X}}$ and it is less than or equal to the number of indecomposable direct summands of $N_{\beta} \oplus \oplus N_{\alpha^{Y}}$, we get

$$
\beta_{i}^{\widetilde{Z}}=\beta_{i}^{X}+\gamma_{i}^{Y}=\beta_{i}^{\widetilde{X}}+\beta_{i}^{\widetilde{Y}}
$$

for all $i=1, \ldots, \min \left\{\overline{\beta_{1}^{X}}, \overline{\beta_{1}^{Y}}\right\}$. By Lemma 5.9 we get:

$$
\sum_{j=1}^{i} \beta_{j}^{Z} \leq \sum_{j=1}^{i}\left(\beta_{j}^{\widetilde{X}}+\beta_{j}^{\widetilde{Y}}\right)=\sum_{j=1}^{i} \beta_{j}^{\widetilde{Z}},
$$

for all $i=1, \ldots, \min \left\{\overline{\beta_{1}^{X}}, \overline{\beta_{1}^{Y}}\right\}$. If $\overline{\beta_{1}^{X}}=\overline{\beta_{1}^{Y}}$, we are done. 
Assume that $\overline{\beta_{1}^{X}}>\overline{\beta_{1}^{Y}}$ and $i>m=\min \left\{\overline{\beta_{1}^{X}}, \overline{\beta_{1}^{Y}}\right\}$. In this case $\beta_{i}^{Y}=$ $\gamma_{i}^{Y}=0$. By Steps 4 and 5 of Algorithm 5.2, we get $\beta_{i}^{\widetilde{Z}}=\beta_{i}^{\widetilde{X}}$. Lemma 5.9 and the inequalities given just above imply:

$$
\begin{aligned}
\sum_{j=1}^{i} \beta_{j}^{Z} & \leq \sum_{j=1}^{i}\left(\beta_{j}^{\widetilde{X}}+\beta_{j}^{\widetilde{Y}}\right) \leq \sum_{j=1}^{m} \beta_{j}^{\widetilde{Z}}+\sum_{j=m+1}^{i}\left(\beta_{j}^{\widetilde{X}}+\beta_{j}^{\widetilde{Y}}\right)= \\
& =\sum_{j=1}^{m} \beta_{j}^{\widetilde{Z}}+\sum_{j=m+1}^{i} \beta_{j}^{\widetilde{X}}=\sum_{j=1}^{m} \beta_{j}^{\widetilde{Z}}+\sum_{j=m+1}^{i} \beta_{j}^{\widetilde{Z}}
\end{aligned}
$$

for all $i$ and we are done.

Assume that $\overline{\beta_{1}^{Y}}>\overline{\beta_{1}^{X}}$. For $i>m=\min \left\{\overline{\beta_{1}^{X}}, \overline{\beta_{1}^{Y}}\right\}$, by Step 4 of Algorithm 5.2, we have

$$
\beta_{i}^{\widetilde{Z}}=\beta_{i}^{Y}+\mathbf{1}\left\{\gamma_{Y}^{i}=\beta_{Y}^{i} \text { and } n>0\right\}
$$

Consider two cases:

- Assume that $n>0$ for $i$. Note that $n>0$ for all $m \leq j<i$. Steps 4 and 5 of Algorithm 5.2 gives

$$
\beta_{i}^{\widetilde{Z}}=\gamma_{i}^{Y}+1
$$

because for $\gamma_{i}^{Y} \neq \beta_{i}^{Y}$ we have $\beta_{i}^{Y}=\gamma_{i}^{Y}+1$ (we are working in category $\mathcal{S}_{1}$ ) and otherwise $\mathbf{1}\left\{\gamma_{Y}^{i}=\beta_{Y}^{i}\right.$ and $\left.n>0\right\}=1$. By the definition of $\widetilde{X}$ and $\widetilde{Y}$ we get

$$
\gamma_{i}^{Y}+1=\beta_{i}^{\tilde{Y}}+\beta_{i}^{\tilde{X}}
$$

and by 5.11 we get $\beta_{j}^{\widetilde{Z}}=\beta_{j}^{\widetilde{Y}}+\beta_{j}^{\widetilde{X}}$ for all $j \leq i$. By Lemma 5.9 applied to partitions $\beta^{\widetilde{Y}}, \beta^{\widetilde{X}}, \beta^{Z}$ we obtain:

$$
\sum_{j=1}^{i} \beta_{j}^{Z} \leq \sum_{j=1}^{i}\left(\beta_{j}^{\widetilde{X}}+\beta_{j}^{\widetilde{Y}}\right)=\sum_{j=1}^{i} \beta_{j}^{\widetilde{Z}} .
$$

- Assume that $n=0$ for $i$. Applying Steps 3 and 4 of Algorithm 5.2, it is easy to deduce that

$$
\sum_{j=1}^{i} \beta_{j}^{\widetilde{Z}}=\sum_{j=1}^{i} \beta_{j}^{X}+\sum_{j=1}^{i} \beta_{j}^{Y} .
$$


By Lemma 5.9 applied to partitions $\beta^{Y}, \beta^{X}$ and $\beta^{Z}$ we obtain:

$$
\sum_{j=1}^{i} \beta_{j}^{Z} \leq \sum_{j=1}^{i} \beta_{j}^{X}+\sum_{j=1}^{i} \beta_{j}^{Y}=\sum_{j=1}^{i} \beta_{j}^{\widetilde{Z}} .
$$

Corollary 5.12. Let $K$ be an algebraically closed field. The extension $Y * X$ computed by Algorithm 5.2 is the generic extension of $Y$ by $X$.

Proof. By Lemma 5.10 any extension $Z$ of $Y$ by $X$ satisfies $Y * X \leq_{\text {dom }} Z$ and hence $Y * X \leq_{\operatorname{deg}} Z$, see Theorem 4.4. It follows that $\mathcal{O}_{Z} \subseteq \overline{\mathcal{O}}_{Y * X}$. Therefore any extension $Z$ of $Y$ by $X$ that it not isomorphic to $Y * X$ satisfies $\operatorname{dim} \mathcal{O}_{Z}<$ $\operatorname{dim} \mathcal{O}_{Y * X}$ and $\operatorname{dim}_{K} \operatorname{End}_{\mathcal{S}}(Z)>\operatorname{dim}_{K} \operatorname{End}_{\mathcal{S}}(Y * X)$ (by the formula for the dimension of an orbit). We are done.

\section{Properties of generic extensions}

In Section 6.1 we prove that the operation $*$ of taking the generic extension provides the set of isomorphism classes of objects in $\mathcal{S}_{1}$ in an associative monoid structure (Lemma 6.4) and we describe generators of this monoid (Lemma 6.6). For this we need Lemma 6.2 (which is a key lemma in this section and it is also used in the proof of Theorem 1.1). We start with a technical fact that one can easily deduce analyzing Algorithm 5.2.

Lemma 6.1. Let $X, Y \in \mathcal{S}_{1}$ be defined by $\left(\gamma^{X}, \beta^{X}\right)$ and $\left(\gamma^{Y}, \beta^{Y}\right)$, respectively. Algorithm 5.2 constructs the object $Z=Y * X$ having the following properties

(1) $\beta_{i}^{Z}=\beta_{i}^{X}+\gamma_{i}^{Y}$, for $i=1, \ldots, \overline{\beta_{1}^{X}}$,

(2) $\sum_{i=1}^{k} \beta_{i}^{Y * X}=\sum_{i=1}^{k}\left(\gamma_{i}^{Y}+\beta_{i}^{X}\right)+\min \left\{k-\overline{\beta_{1}^{X}}, \sum_{i=1}^{k}\left(\beta_{i}^{Y}-\gamma_{i}^{Y}\right)\right\}$, for all

Proof. The statement (1) follows from the Steps 3 and 4 of Algorithm 5.2 and the fact that $\beta_{i}^{Y}=\gamma_{i}^{Y}=0$ for $i=\min \left\{\overline{\beta_{1}^{X}}, \overline{\beta_{1}^{Y}}\right\}+1, \ldots, \overline{\beta_{1}^{X}}$. 
The statement $(2)$ we prove inductively. We have only consider the case $\overline{\beta_{1}^{Y}}>\overline{\beta_{1}^{X}}$. Let $k=\overline{\beta_{1}^{X}}+1$. We have $\beta_{k}^{X}=0$ and

$$
\sum_{i=1}^{k} \beta_{i}^{Y * X}=\sum_{i=1}^{\overline{\beta_{1}^{X}}}\left(\gamma_{i}^{Y}+\beta_{i}^{X}\right)+\beta_{k}^{Y}+\mathbf{1}\left\{\gamma_{k}^{Y}=\beta_{k}^{Y} \text { and } n>0\right\}
$$

Note that

$\min \left\{k-\overline{\beta_{1}^{X}}, \sum_{i=1}^{k}\left(\beta_{i}^{Y}-\gamma_{i}^{Y}\right)\right\}=\min \left\{1, n+\beta_{k}^{Y}-\gamma_{k}^{Y}\right\}= \begin{cases}1 & \text { if } n>0 \text { or } \beta_{k}^{Y}>\gamma_{k}^{Y} \\ 0 & \text { otherwise }\end{cases}$

It follows that $\gamma_{k}^{Y}+\beta_{k}^{X}+\min \left\{1, n+\beta_{k}^{Y}-\gamma_{k}^{Y}\right\}=\beta_{k}^{Y}+\mathbf{1}\left\{\gamma_{k}^{Y}=\beta_{k}^{Y}\right.$ and $\left.n>0\right\}$. In the induction step we apply similar arguments.

Lemma 6.2. Let $Y, Y^{\prime} \in \mathcal{S}_{a}^{b}$ for some natural numbers $a, b$ and let $Y \leq_{\operatorname{deg}} Y^{\prime}$. For any $X \in \mathcal{S}_{1}$ following condition holds:

1. $Y * X \leq_{\operatorname{deg}} Y^{\prime} * X$,

2. $X * Y \leq_{\operatorname{deg}} X * Y^{\prime}$.

Proof. Let $Y, Y^{\prime} \in \mathcal{S}_{a}^{b}$ and $Y \leq_{\operatorname{deg}} Y^{\prime}$. By Theorem 4.4 the inequality $Y \leq_{\text {dom }}$ $Y^{\prime}$ holds. Therefore for any natural $k$ we have

$$
\sum_{i=1}^{k} \lambda_{i}^{Y} \geq \sum_{i=1}^{k} \lambda_{i}^{Y^{\prime}}
$$

where $\lambda \in\{\beta, \gamma\}$. By Step 1 of Algorithm 5.2 we have $\gamma^{Y * X}=\gamma^{X}+\gamma^{Y}$. Therefore

$$
\sum_{i=1}^{k} \gamma_{i}^{Y * X}=\sum_{i=1}^{k}\left(\gamma_{i}^{Y}+\gamma_{i}^{X}\right) \geq \sum_{i=1}^{k}\left(\gamma_{i}^{Y^{\prime}}+\gamma_{i}^{X}\right)=\sum_{i=1}^{k} \gamma_{i}^{Y^{\prime} * X}
$$

for any $k$. It follows that $\gamma^{Y * X} \leq_{\text {nat }} \gamma^{Y^{\prime} * X}$ and similarly $\gamma^{X * Y} \leq_{\text {nat }} \gamma^{X * Y^{\prime}}$.

By Lemma 6.1 and the inequality 6.3, for $k \leq \overline{\beta_{1}^{X}}$ we have

$$
\sum_{i=1}^{k} \beta_{i}^{Y * X}=\sum_{i=1}^{k}\left(\gamma_{i}^{Y}+\beta_{i}^{X}\right) \geq \sum_{i=1}^{k}\left(\gamma_{i}^{Y^{\prime}}+\beta_{i}^{X}\right)=\sum_{i=1}^{k} \beta_{i}^{Y^{\prime} * X}
$$


and for $k>\overline{\beta_{1}^{X}}$

$$
\sum_{i=1}^{k} \beta_{i}^{Y * X}=\sum_{i=1}^{k}\left(\gamma_{i}^{Y}+\beta_{i}^{X}\right)+\min \left\{k-\bar{\beta}_{1}^{X}, \sum_{i=1}^{k}\left(\beta_{i}^{Y}-\gamma_{i}^{Y}\right)\right\}
$$

If $\min \left\{k-\overline{\beta_{1}^{X}}, \sum_{i=1}^{k}\left(\beta_{i}^{Y}-\gamma_{i}^{Y}\right)\right\}=k-\bar{\beta}_{1}^{X}$ then

$$
\begin{aligned}
\sum_{i=1}^{k} \beta_{i}^{Y * X}= & \sum_{i=1}^{k}\left(\gamma_{i}^{Y}+\beta_{i}^{X}\right)+k-\bar{\beta}_{1}^{X} \geq \sum_{i=1}^{k}\left(\gamma_{i}^{Y^{\prime}}+\beta_{i}^{X}\right)+k-\bar{\beta}_{1}^{X} \geq \\
& \sum_{i=1}^{k}\left(\gamma_{i}^{Y^{\prime}}+\beta_{i}^{X}\right)+\min \left\{k-\bar{\beta}_{1}^{X}, \sum_{i=1}^{k}\left(\beta_{i}^{Y^{\prime}}-\gamma_{i}^{Y^{\prime}}\right)\right\}=\sum_{i=1}^{k} \beta_{i}^{Y^{\prime} * X}
\end{aligned}
$$

otherwise

$$
\begin{aligned}
\sum_{i=1}^{k} \beta_{i}^{Y * X}= & \sum_{i=1}^{k}\left(\gamma_{i}^{Y}+\beta_{i}^{X}\right)+\sum_{i=1}^{k}\left(\beta_{i}^{Y}-\gamma_{i}^{Y}\right)=\sum_{i=1}^{k}\left(\beta_{i}^{X}+\beta_{i}^{Y}\right) \geq \\
& \sum_{i=1}^{k}\left(\beta_{i}^{X}+\beta_{i}^{Y^{\prime}}\right)=\sum_{i=1}^{k}\left(\gamma_{i}^{Y^{\prime}}+\beta_{i}^{X}\right)+\sum_{i=1}^{k}\left(\beta_{i}^{Y^{\prime}}-\gamma_{i}^{Y^{\prime}}\right) \geq \\
& \sum_{i=1}^{k}\left(\gamma_{i}^{Y^{\prime}}+\beta_{i}^{X}\right)+\min \left\{k-\bar{\beta}_{1}^{X}, \sum_{i=1}^{k}\left(\beta_{i}^{Y^{\prime}}-\gamma_{i}^{Y^{\prime}}\right)\right\}=\sum_{i=1}^{k} \beta_{i}^{Y^{\prime} * X}
\end{aligned}
$$

Therefore $\beta^{Y * X} \leq_{\text {nat }} \beta^{Y^{\prime} * X}$ and $Y * X \leq_{\text {dom }} Y^{\prime} * X$. Again by Theorem 4.4 we obtain $Y * X \leq_{\operatorname{deg}} Y^{\prime} * X$.

Since $\overline{\beta_{1}^{Y}} \leq \overline{\beta_{1}^{Y^{\prime}}}$ (or equivalently $\beta_{1}^{Y} \geq \beta_{1}^{Y^{\prime}}$ ), in the same way we can see that $X * Y \leq_{\text {dom }} X * Y^{\prime}$ for any $X \in \mathcal{S}$.

\subsection{The monoid of generic extensions}

The associative property of $*$ is established by the next lemma.

Lemma 6.4. If $X, Y, Z \in \mathcal{S}_{1}$, then $X *(Y * Z)=(X * Y) * Z$. 
Proof. We follow the arguments given in [17]. Consider the following diagram

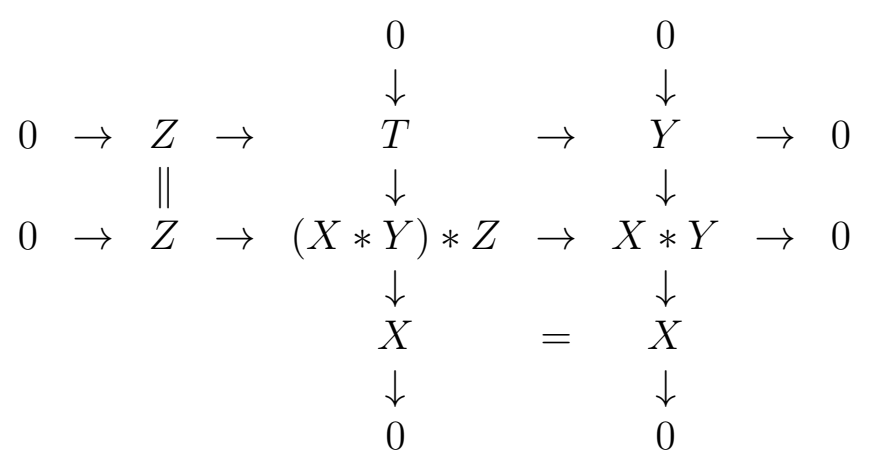

where the top row is a pull-back of the bottom. It follows from Lemma 5.10 that $Y * Z \leq_{\operatorname{deg}} T$ and $X * T \leq_{\operatorname{deg}}(X * Y) * Z$. Therefore by Lemma 6.2 we have $X *(Y * Z) \leq_{\operatorname{deg}}(X * Y) * Z$.

Dually, applying push out we get $(X * Y) * Z \leq_{\operatorname{deg}} X *(Y * Z)$. This finishes the proof (because $\leq_{\text {deg }}$ is a partial order).

Remark 6.5. We can consider the associative monoid

$$
\mathcal{M}=(\mathcal{M},[0], *)
$$

where $\mathcal{M}$ is the set of isomorphism classes of objects in $\mathcal{S}_{1},[0]$ is the isomorphism class of the zero object and $*$ is the operation of taking the generic extensions: $[X] *[Y]=[X * Y]$.

Lemma 6.6. The monoid $\mathcal{M}$ is generated by the set

$$
\left\{\left[P_{1}^{1}\right],\left[\left(P_{0}^{1}\right)^{\oplus n}\right] \text { for } n \geq 1\right\} .
$$

Proof. Note that

- $\left[P_{0}^{m}\right]=\left[P_{0}^{1}\right]^{* m}$ for all $m \geq 1$,

- $\left[P_{1}^{m}\right]=\left[P_{0}^{1}\right]^{*(m-1)} *\left[P_{1}^{1}\right]$ for all $m \geq 1$,

- $\left[\left(P_{1}^{1}\right)^{\oplus m}\right]=\left[P_{1}^{1}\right]^{* m}$ for all $m \geq 1$,

- $\left[\left(P_{0}^{1}\right)^{\oplus n} \oplus\left(P_{1}^{1}\right)^{\oplus m}\right]=\left[P_{1}^{1}\right]^{* m} *\left[P_{0}^{1}\right]^{\oplus n}$ for all $m, n \geq 1$. 
Finally, let $m_{1}, \ldots, m_{s} \geq 2, m_{s+1}=\ldots=m_{t}=1$ and $\varepsilon_{i} \in\{0,1\}$ for all $i=1, \ldots, s+t$. We get

$$
\left[\bigoplus_{i=1}^{s+t} P_{\varepsilon_{i}}^{m_{i}}\right]=\left[\left(P_{0}^{1}\right)^{\oplus s} \oplus \bigoplus_{i=s+1}^{t} P_{\varepsilon_{i}}^{1}\right] *\left[\bigoplus_{i=1}^{s} P_{\varepsilon_{i}}^{m_{i}-1}\right]
$$

By induction we are done.

\section{Generic extensions - geometry}

In this section we present geometric interpretation of generic extensions and we prove Theorem 1.1 .

Let $K$ be an algebraically closed field. Fix subsets $\mathcal{Y} \subseteq{ }^{a} V^{b}(K), \mathcal{X} \subseteq$ ${ }^{c} V^{d}(K)$ and $Y=(f, \varphi) \in \mathcal{Y}, X=(g, \phi) \in \mathcal{X}$. Let

$Z(Y, X)=\left\{(h, \nu) \in \mathbb{M}_{d \times a} \times \mathbb{M}_{d \times b} ;\left(\left[\begin{array}{ll}g & h \\ 0 & f\end{array}\right],\left[\begin{array}{cc}\phi & \nu \\ 0 & \varphi\end{array}\right]\right) \in{ }^{a+c} V^{b+d}(K)\right\}$

and

$$
\begin{aligned}
& \mathcal{Z}(\mathcal{Y}, \mathcal{X})=\left\{\left(\left[\begin{array}{ll}
g & h \\
0 & f
\end{array}\right],\left[\begin{array}{ll}
\phi & \nu \\
0 & \varphi
\end{array}\right]\right)\right. \\
&Y=(f, \varphi) \in \mathcal{Y}, X=(g, \phi) \in \mathcal{X},(h, \nu) \in Z(Y, X)\} .
\end{aligned}
$$

Lemma 7.1. If the sets $\mathcal{Y} \subseteq{ }^{a} V^{b}, \mathcal{X} \subseteq{ }^{c} V^{d}$ are closed, then the set $\mathcal{Z}(\mathcal{Y}, \mathcal{X}) \subseteq{ }^{a+c} V^{b+d}$ is closed.

Proof. Since the sets $\mathcal{Y} \subseteq{ }^{a} V^{b}, \mathcal{X} \subseteq{ }^{c} V^{d}$ are closed (in the Zariski topology), there exists a finite set of equations deciding whether $Y=(f, \varphi) \in \mathcal{Y}, X=$ $(g, \phi) \in \mathcal{X}$. If $f, g$ have maximal rank, then $\left[\begin{array}{cc}g & h \\ 0 & f\end{array}\right]$ has also maximal rank. Now, it is easy to see that there exists a finite set of equations deciding whether an element belongs to the set $\mathcal{Z}(\mathcal{Y}, \mathcal{X})$. It follows that the set $\mathcal{Z}(\mathcal{Y}, \mathcal{X}) \subseteq{ }^{a+c} V^{b+d}$ is closed.

Let

$$
\mathcal{E}(\mathcal{Y}, \mathcal{X})=\operatorname{Gl}(a+c, b+d) \cdot \mathcal{Z}(\mathcal{Y}, \mathcal{X}) \subseteq{ }^{a+c} V^{b+d}
$$

Note that $\mathcal{E}(\mathcal{Y}, \mathcal{X})$ is the set of all extensions $Z$ of some $Y \in \mathcal{Y}$ by some $X \in \mathcal{X}$, i.e. there exists a short exact sequence

$$
0 \rightarrow X \rightarrow Z \rightarrow Y \rightarrow 0 \text {. }
$$


Lemma 7.2. Let $Y \in{ }^{a} V^{b}, X \in{ }^{c} V^{d}$. The set $\mathcal{E}\left(\overline{\mathcal{O}_{Y}}, \overline{\mathcal{O}_{X}}\right)$ is closed in ${ }^{a+c} V^{b+d}$.

Proof. The set $\mathcal{Z}\left(\overline{\mathcal{O}_{Y}}, \overline{\mathcal{O}_{X}}\right)$ is stable under the action of the parabolic subgroup

$$
\left[\begin{array}{cc}
\operatorname{Gl}(a) & * \\
0 & \mathrm{Gl}(c)
\end{array}\right] \times\left[\begin{array}{cc}
\mathrm{Gl}(b) & * \\
0 & \mathrm{Gl}(d)
\end{array}\right]
$$

of the group $\operatorname{Gl}(a+c, b+d)$. Moreover, by Lemma 7.1, the set $\mathcal{Z}\left(\overline{\mathcal{O}_{Y}}, \overline{\mathcal{O}_{X}}\right)$ is closed. Therefore by [14, Proposition 6.6] the set $\mathcal{E}\left(\overline{\mathcal{O}_{Y}}, \overline{\mathcal{O}_{X}}\right)=\operatorname{Gl}(a+c, b+$ $d) \cdot \mathcal{Z}\left(\overline{\mathcal{O}_{Y}}, \overline{\mathcal{O}_{X}}\right)$ is closed.

Proof of Theorem 1.1. Let $Y \in{ }^{a} V^{b}, X \in{ }^{c} V^{d}$.

(1) and (2) By the classification of objects in $\mathcal{S}_{1}$, recalled in Section 2.2, it follows that $\mathcal{E}\left(\mathcal{O}_{Y}, \mathcal{O}_{X}\right)$ is a union of finitely many orbits $\mathcal{O}_{Z}$ corresponding to extensions $Z$ of $Y$ by $X$. By Lemmma 5.10 and Theorem 4.4 the object $Y * X$ computed by Algorithm 5.2 is the unique extension of $Y$ by $X$ that is minimal in the degeneration order. Therefore $\mathcal{O}_{Z} \subseteq \overline{\mathcal{O}_{Y * X}}$ for any extension $Z$ of $Y$ by $X$. It follows that $\mathcal{O}_{Y * X}$ is the unique dense orbit in $\mathcal{E}\left(\mathcal{O}_{Y}, \mathcal{O}_{X}\right)$. As an immediate consequence we get $\overline{\mathcal{O}_{Y * X}}=\overline{\mathcal{E}\left(\mathcal{O}_{Y}, \mathcal{O}_{X}\right)}$.

(3) We prove that $\mathcal{E}\left(\overline{\mathcal{O}_{Y}}, \overline{\mathcal{O}_{X}}\right) \subseteq \overline{\mathcal{O}_{Y * X}}$. Let $Z$ be an extension of $Y^{\prime}$ by $X^{\prime}$ such that $Y^{\prime} \in \overline{\mathcal{O}_{Y}}$ and $X^{\prime} \in \overline{\mathcal{O}_{X}}$. It means that $Y \leq_{\operatorname{deg}} Y^{\prime}$ and $X \leq_{\operatorname{deg}} X^{\prime}$. By Lemma 6.2 and (1), we have $Y * X \leq_{\operatorname{deg}} Y^{\prime} * X^{\prime} \leq_{\operatorname{deg}} Z$. Therefore $Z \in \overline{\mathcal{O}_{Y * X}}$.

Consequently, we have $\mathcal{E}\left(\mathcal{O}_{Y}, \mathcal{O}_{X}\right) \subseteq \mathcal{E}\left(\overline{\mathcal{O}_{Y}}, \overline{\mathcal{O}_{X}}\right) \subseteq \overline{\mathcal{O}_{Y * X}}$. Since the set $\mathcal{E}\left(\overline{\mathcal{O}_{Y}}, \overline{\mathcal{O}_{X}}\right)$ is closed and $\overline{\mathcal{O}_{Y * X}}=\overline{\mathcal{E}\left(\mathcal{O}_{Y}, \mathcal{O}_{X}\right)}$, we get $\overline{\mathcal{E}\left(\mathcal{O}_{Y}, \mathcal{O}_{X}\right)}=$ $\mathcal{E}\left(\overline{\mathcal{O}_{Y}}, \overline{\mathcal{O}_{X}}\right)=\overline{\mathcal{O}_{Y * X}}$.

(4) Since the group $\operatorname{Gl}(a, b)$ is irreducible for all $a, b$, the sets $\mathcal{O}_{X}, \overline{\mathcal{O}_{X}}$ are irreducible for all $X$. By (3) we are done.

\section{References}

[1] I. Assem, D. Simson oraz A. Skowroński, "Elements of the Representation Theory of Associative Algebras", Vol. I: Techniques of Representation Theory, London Mathematical Society Student Texts, 65. Cambridge University Press, Cambridge, 2006.

[2] D. Beers, R. Hunter, E. Walker, Finite valuated p-groups, Abelian Group Theory. LNM 1006, Springer (1983), 471-506. 
[3] K. Bongartz, Degenerations for representations of tame quivers, Ann. Scient. Éc. Norm. Sup. 28 (1995), 647-668.

[4] K. Bongartz, On degenerations and extensions of finite dimensional modules, Adv. Math. 121 (1996), 245-287.

[5] B. Deng and J. Du, Monomial bases for quantum affine $\mathfrak{s l}_{n}$, Adv. Math. 191 (2005), 276-304.

[6] B. Deng, J. Du and A. Mah, Presenting degenerate Ringel-Hall algebras of cyclic quivers, J. Pure Appl. Algebra 214 (2010), 1787-1799.

[7] A. Hubery, The composition algebra and composition monoid of the Kronecker quiver, J. London Math. Soc. 72 (2005) 137-150.

[8] M. Kaniecki, J. Kosakowska, M. Schmidmeier, Operations on arc diagrams and degenerations for invariant subspaces of linear operators. Part II, Comm. Alg. 46 (2018), 2243-2263.

[9] T. Klein, The multiplication of Schur-functions and extensions of $p$ modules, J. Lond. Math. Soc. 43 (1968), 280-284.

[10] J. Kosakowska, Generic extensions of prinjective modules, Algebras and Representations Theory, (2006) 9: 557-568.

[11] J. Kosakowska, Generic extensions of nilpotent $K[T]$-modules, monoids of partitions and constant terms of Hall polynomials, Coll. Math. 128 (2012), 253-261.

[12] J. Kosakowska and M. Schmidmeier, Operations on arc diagrams and degenerations for invariant subspaces of linear operators, Trans. Amer. Math. Soc. 367 (2015), 5475-5505.

[13] J. Kosakowska and M. Schmidmeier, The boundary of the irreducible components for invariant subspace varieties, Math. Zeit. 290 (2018), 953-972.

[14] H. Kraft, Geometric methods in representation theory, In: Representations of Algebras. Workshop Proceedings, Puebla, Mexico (1980). Lecture Notes in Mathematics, Vol. 944 (Springer-Verlag, Berlin, Heilderberg, New York, Tokyo 1982). 
[15] I. G. Macdonald, Symmetric Functions and Hall Polynomials, Oxford University Press, 1995.

[16] M. Reineke, Hall-Algebren, Quantengruppen und Lusztigs kanonische Basis, Diplomarbeit, Bergische Universität - Gesamthochschule Wuppertal, 1993/94.

[17] M. Reineke, Generic extensions and multiplicative bases of quantum groups at $q=0$, Represent. Theory 5 (2001), 147-163.

[18] C. Riedtmann, Degenerations for representations of quiver with relations, Ann. Sci. Ec. Norm. Super. 4 (1986), 275-301.

[19] M. Schmidmeier, Bounded submodules of modules, J. Pure Appl. Algebra 203 (2005), 45-82.

[20] M. Schmidmeier, Hall polynomials via automorphisms of short exact sequences, Algebr. Represent. Theory (15) (2012), 449-481.

[21] G. Zwara, Degenerations for modules over representation-finite biserial algebras, J. Algebra 198 (1997), 563-581. 